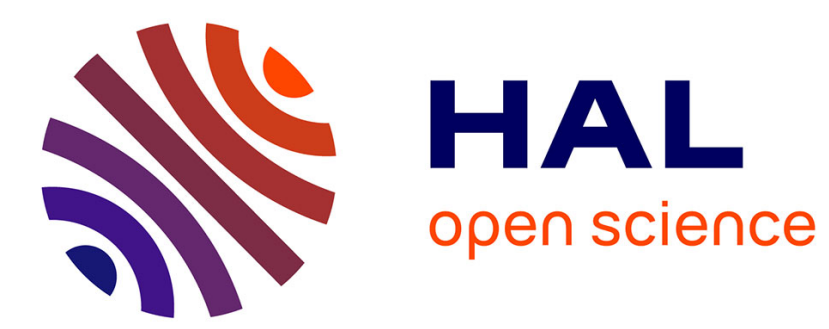

\title{
An existence and uniqueness theorem for the dynamics of flexural shells
}

Paolo Piersanti

\section{To cite this version:}

Paolo Piersanti. An existence and uniqueness theorem for the dynamics of flexural shells. 2019. hal-02135023v3

\section{HAL Id: hal-02135023 \\ https://hal.science/hal-02135023v3}

Preprint submitted on 27 Aug 2019

HAL is a multi-disciplinary open access archive for the deposit and dissemination of scientific research documents, whether they are published or not. The documents may come from teaching and research institutions in France or abroad, or from public or private research centers.
L'archive ouverte pluridisciplinaire HAL, est destinée au dépôt et à la diffusion de documents scientifiques de niveau recherche, publiés ou non, émanant des établissements d'enseignement et de recherche français ou étrangers, des laboratoires publics ou privés. 


\title{
An existence and uniqueness theorem for the dynamics of flexural shells
}

\author{
Journal Title \\ XX(X):1-26 \\ (C) The Author(s) 0000 \\ Reprints and permission \\ sagepub.co.uk/journalsPermissions.nav \\ DOI: $10.1177 /$ ToBeAssigned \\ www.sagepub.com / \\ @SAGE
}

\section{Paolo Piersanti ${ }^{1,2}$}

\begin{abstract}
In this paper we define a priori what is a natural two-dimensional model for a timedependent flexural shell. As expected, this model takes the form of a set of hyperbolic variational equations posed over the space of admissible linearized inextensional displacements, and a set of initial conditions. Using a classical argument, we prove that the model under consideration admits a unique strong solution. However, the latter strategy makes use of function spaces which are not amenable for numerically approximating the solution. We thus provide an alternate formulation of the studied problem using a suitable penalty scheme, which is more suitable in the context of numerical approximations. For sake of completeness, in the final part of the paper, we also provide an existence and uniqueness theorem in the case where the linearly elastic shell under consideration is an elliptic membrane shell.
\end{abstract}

\section{Keywords}

Linearly elastic flexural shells, hyperbolic equations, penalty method, constrained optimization, Galerkin method

\footnotetext{
${ }^{1}$ Department of Mathematics, City University of Hong Kong, 83 Tat Chee Avenue, Kowloon, Hong Kong

2 Institute of Mathematics and Scientific Computing, Karl-Franzens-Universitat Graz, 36 Heinrichstrasse, Graz, Austria

Corresponding author:

Paolo Piersanti, Department of Mathematics, City University of Hong Kong, 83 Tat Chee Avenue, Kowloon, Hong Kong

Paolo Piersanti, Institute of Mathematics and Scientific Computing, Karl-Franzens-Universitat Graz, 36 Heinrichstrasse, Graz, Austria

Email: ppiersan-c@my.cityu.edu.hk paolo.piersanti@uni-graz.at

Prepared using sagej.cls [Version: 2016/06/24 v1.10]
} 


\section{Introduction}

Flexural shells are widely used in many applicative fields such as physics, engineering and material science. Some remarkable applications involving the usage of such shells are: reinforced oil palm shell and palm oil clinker concrete (PSCC) beam [1], smart composite shell panels [2], functionally graded spherical shell panel [3], anisogrid lattice conical shells [4], and reinforced Eco-friendly coconut shell concrete [5]. Because of its wide range of applications, the theory of flexural shells is one of the most important branches in Mathematical Elasticity.

Unlike the static case, which was addressed by Ciarlet and his associates in the references [6], [7], [8], [9], [10], [11], [12], [13], [14], [15], [16], [17], [18], there are very few reference about the time-dependent case. In this direction we cite, for instance, the papers [19] and [20].

To our best knowledge, there are no references that treat the existence and uniqueness of solutions for the dynamics of flexural shells with mathematical rigour.

In Section 2 we present some geometrical and analytical background; in Section 3 we formulate the problem describing the displacement of a flexural shell when it is subjected to a dynamic load; in Section 4 we prove the sought existence and uniqueness result by relying on classical arguments that, however, are not amenable for the implementation of a suitable numerical scheme; in Sections 5 and 6 we prove the existence and uniqueness of solutions using a penalty scheme, which is easier to treat in a context of numerical simulations; finally, in Section 7, we provide an existence and uniqueness theorem in the case where the linearly elastic shell under consideration is an elliptic membrane shell.

\section{Geometrical preliminaries}

For details about the classical notions of differential geometry recalled in this section see, e.g., [21] or [22].

Greek indices, except $\varepsilon$ and $\nu$, take their values in the set $\{1,2\}$, while Latin indices, except when they are used for indexing sequences, take their values in the set $\{1,2,3\}$, and the summation convention with respect to repeated indices is systematically used in conjunction with these two rules. The notation $\mathbb{E}^{3}$ designates the three-dimensional Euclidean space; the Euclidean inner product and the vector product of $\boldsymbol{u}, \boldsymbol{v} \in \mathbb{E}^{3}$ are denoted $\boldsymbol{u} \cdot \boldsymbol{v}$ and $\boldsymbol{u} \wedge \boldsymbol{v}$; the Euclidean norm of $\boldsymbol{u} \in \mathbb{E}^{3}$ is denoted $|\boldsymbol{u}|$. The notation $\delta_{i}^{j}$ designates the Kronecker symbol.

Given an open subset $\Omega$ of $\mathbb{R}^{n}$, notations such as $L^{2}(\Omega), H^{m}(\Omega)$, or $H_{0}^{m}(\Omega)$, $m \geq 1$, designate the usual Lebesgue and Sobolev spaces, and the notation $\mathcal{D}(\Omega)$ designates the space of all functions that are infinitely differentiable over $\Omega$ and have compact support in $\Omega$. The notation $\|\cdot\|_{X}$ designates the norm in a normed vector space $X$. The dual space of a vector space $X$ is denoted by $X^{*}$ and the duality pair between $X^{*}$ and $X$ is denoted by $X^{*}\langle\cdot, \cdot\rangle_{X}$. Spaces of vector-valued functions are denoted with boldface letters. Lebesgue-Bochner spaces defined over a bounded open interval $I$ (cf. [23]), are denoted $L^{p}(I ; H)$, where $H$ is a Banach space and $1 \leq p \leq \infty$. The notation $\|\cdot\|_{0, \Omega}$ designates the norm of the

Prepared using sagej.cls 
Lebesgue space $L^{2}(\Omega)$, and the notation $\|\cdot\|_{m \Omega}$, designates the norm of the Sobolev space $H^{m}(\Omega), m \geq 1$. The notation $\|\cdot\|_{L^{p}(I ; H)}$ designates the norm of the Lebesgue-Bochner space $L^{p}(I ; H)$. The notations $\dot{\eta}$ and $\ddot{\eta}$ denote the first weak derivative with respect to $t \in I$ and second weak derivative with respect to $t \in I$ of a scalar function $\eta$ defined over the interval $I$. The notations $\dot{\boldsymbol{\eta}}$ and $\ddot{\boldsymbol{\eta}}$ denote the first weak derivative with respect to $t \in I$ and second weak derivative with respect to $t \in I$ of a vector-valued function $\boldsymbol{\eta}$ defined over the interval $I$.

A domain in $\mathbb{R}^{n}$ is a bounded and connected open subset $\Omega$ of $\mathbb{R}^{n}$, whose boundary $\partial \Omega$ is Lipschitz-continuous, the set $\Omega$ being locally on a single side of $\partial \Omega$.

Let $\omega$ be a domain in $\mathbb{R}^{2}$, let $y=\left(y_{\alpha}\right)$ denote a generic point in $\omega$, and let $\partial_{\alpha}:=\partial / \partial y_{\alpha}$ and $\partial_{\alpha \beta}:=\partial^{2} / \partial y_{\alpha} \partial y_{\beta}$. A mapping $\boldsymbol{\theta} \in \mathcal{C}^{1}\left(\bar{\omega} ; \mathbb{E}^{3}\right)$ is an immersion if the two vectors

$$
\boldsymbol{a}_{\alpha}(y):=\partial_{\alpha} \boldsymbol{\theta}(y)
$$

are linearly independent at each point $y \in \bar{\omega}$. Then the image $\boldsymbol{\theta}(\bar{\omega})$ of the set $\bar{\omega}$ under the mapping $\boldsymbol{\theta}$ is a surface in $\mathbb{E}^{3}$, equipped with $y_{1}, y_{2}$ as its curvilinear coordinates. Given any point $y \in \bar{\omega}$, the vectors $\boldsymbol{a}_{\alpha}(y)$ span the tangent plane to the surface $\boldsymbol{\theta}(\bar{\omega})$ at the point $\boldsymbol{\theta}(y)$, the unit vector

$$
\boldsymbol{a}_{3}(y):=\frac{\boldsymbol{a}_{1}(y) \wedge \boldsymbol{a}_{2}(y)}{\left|\boldsymbol{a}_{1}(y) \wedge \boldsymbol{a}_{2}(y)\right|}
$$

is normal to $\boldsymbol{\theta}(\bar{\omega})$ at $\boldsymbol{\theta}(y)$, the three vectors $\boldsymbol{a}_{i}(y)$ form the covariant basis at $\boldsymbol{\theta}(y)$, and the three vectors $\boldsymbol{a}^{j}(y)$ defined by the relations

$$
\boldsymbol{a}^{j}(y) \cdot \boldsymbol{a}_{i}(y)=\delta_{i}^{j}
$$

form the contravariant basis at $\boldsymbol{\theta}(y)$; note that the vectors $\boldsymbol{a}^{\beta}(y)$ also span the tangent plane to $\boldsymbol{\theta}(\bar{\omega})$ at $\boldsymbol{\theta}(y)$ and that $\boldsymbol{a}^{3}(y)=\boldsymbol{a}_{3}(y)$.

The first fundamental form of the surface $\boldsymbol{\theta}(\bar{\omega})$ is defined by means of its covariant components

$$
a_{\alpha \beta}:=\boldsymbol{a}_{\alpha} \cdot \boldsymbol{a}_{\beta}=a_{\beta \alpha} \in \mathcal{C}^{0}(\bar{\omega})
$$

or by means of its contravariant components

$$
a^{\alpha \beta}:=\boldsymbol{a}^{\alpha} \cdot \boldsymbol{a}^{\beta}=a^{\beta \alpha} \in \mathcal{C}^{0}(\bar{\omega}) .
$$

Note that the symmetric matrix field $\left(a^{\alpha \beta}\right)$ is the inverse of the matrix field $\left(a_{\alpha \beta}\right)$, that $\boldsymbol{a}^{\beta}=a^{\alpha \beta} \boldsymbol{a}_{\alpha}$ and $\boldsymbol{a}_{\alpha}=a_{\alpha \beta} \boldsymbol{a}^{\beta}$, and that the area element along $\boldsymbol{\theta}(\bar{\omega})$ is given at each point $\boldsymbol{\theta}(y), y \in \bar{\omega}$, by $\sqrt{a(y)} \mathrm{d} y$, where

$$
a:=\operatorname{det}\left(a_{\alpha \beta}\right) \in \mathcal{C}^{0}(\bar{\omega}) .
$$

Given an immersion $\boldsymbol{\theta} \in \mathcal{C}^{2}\left(\bar{\omega} ; \mathbb{E}^{3}\right)$, the second fundamental form of the surface $\boldsymbol{\theta}(\bar{\omega})$ is defined by means of its covariant components

$$
b_{\alpha \beta}:=\partial_{\alpha} \boldsymbol{a}_{\beta} \cdot \boldsymbol{a}_{3}=-\boldsymbol{a}_{\beta} \cdot \partial_{\alpha} \boldsymbol{a}_{3}=b_{\beta \alpha} \in \mathcal{C}^{0}(\bar{\omega}),
$$

Prepared using sagej.cls 
or by means of its mixed components

$$
b_{\alpha}^{\beta}:=a^{\beta \sigma} b_{\alpha \sigma} \in \mathcal{C}^{0}(\bar{\omega}),
$$

and the Christoffel symbols associated with the immersion $\boldsymbol{\theta}$ are defined by

$$
\Gamma_{\alpha \beta}^{\sigma}:=\partial_{\alpha} \boldsymbol{a}_{\beta} \cdot \boldsymbol{a}^{\sigma}=\Gamma_{\beta \alpha}^{\sigma} \in \mathcal{C}^{0}(\bar{\omega}) .
$$

The Gaussian curvature at each point $\boldsymbol{\theta}(y), y \in \bar{\omega}$, of the surface $\boldsymbol{\theta}(\bar{\omega})$ is defined by

$$
\kappa(y):=\frac{\operatorname{det}\left(b_{\alpha \beta}(y)\right)}{\operatorname{det}\left(a_{\alpha \beta}(y)\right)}=\operatorname{det}\left(b_{\alpha}^{\beta}(y)\right)
$$

(the denominator in the above relation does not vanish since $\boldsymbol{\theta}$ is assumed to be an immersion). Note that the Gaussian curvature $\kappa(y)$ at the point $\boldsymbol{\theta}(y)$ is also equal to the product of the two principal curvatures at this point.

A surface $\boldsymbol{\theta}(\bar{\omega})$ defined by means of an immersion $\boldsymbol{\theta} \in \mathcal{C}^{2}\left(\bar{\omega} ; \mathbb{E}^{3}\right)$ is said to be elliptic if its Gaussian curvature is everywhere $>0$ in $\bar{\omega}$, or equivalently, if there exists a constant $\kappa_{0}$ such that

$$
0<\kappa_{0} \leq \kappa(y) \quad \text { for all } y \in \bar{\omega} .
$$

Given an immersion $\boldsymbol{\theta} \in \mathcal{C}^{2}\left(\bar{\omega} ; \mathbb{E}^{3}\right)$ and a vector field $\boldsymbol{\eta}=\left(\eta_{i}\right) \in \mathcal{C}^{1}\left(\bar{\omega} ; \mathbb{R}^{3}\right)$, the vector field

$$
\tilde{\boldsymbol{\eta}}:=\eta_{i} \boldsymbol{a}^{i}
$$

can be viewed as a displacement field of the surface $\boldsymbol{\theta}(\bar{\omega})$, thus defined by means of its covariant components $\eta_{i}$ over the vectors $\boldsymbol{a}^{i}$ of the contravariant bases along the surface. If the norms $\left\|\eta_{i}\right\|_{\mathcal{C}^{1}(\bar{\omega})}$ are small enough, the mapping $\left(\boldsymbol{\theta}+\eta_{i} \boldsymbol{a}^{i}\right) \in \mathcal{C}^{1}\left(\bar{\omega} ; \mathbb{E}^{3}\right)$ is also an immersion, so that the set $\left(\boldsymbol{\theta}+\eta_{i} \boldsymbol{a}^{i}\right)(\bar{\omega})$ is also a surface in $\mathbb{E}^{3}$, equipped with the same curvilinear coordinates as those of the surface $\boldsymbol{\theta}(\bar{\omega})$, called the deformed surface corresponding to the displacement field $\tilde{\boldsymbol{\eta}}=\eta_{i} \boldsymbol{a}^{i}$. One can then define the first fundamental form of the deformed surface by means of its covariant components

$$
a_{\alpha \beta}(\boldsymbol{\eta}):=\left(\boldsymbol{a}_{\alpha}+\partial_{\alpha} \tilde{\boldsymbol{\eta}}\right) \cdot\left(\boldsymbol{a}_{\beta}+\partial_{\beta} \tilde{\boldsymbol{\eta}}\right),
$$

and the second fundamental form of the deformed surface by means of its covariant components

$$
b_{\alpha \beta}(\boldsymbol{\eta}):=\partial_{\alpha}\left(\boldsymbol{a}_{\beta}+\partial_{\beta} \tilde{\boldsymbol{\eta}}\right) \cdot \frac{\left(\boldsymbol{a}_{1}+\partial_{1} \tilde{\boldsymbol{\eta}}\right) \wedge\left(\boldsymbol{a}_{2}+\partial_{2} \tilde{\boldsymbol{\eta}}\right)}{\left|\left(\boldsymbol{a}_{1}+\partial_{1} \tilde{\boldsymbol{\eta}}\right) \wedge\left(\boldsymbol{a}_{2}+\partial_{2} \tilde{\boldsymbol{\eta}}\right)\right|}
$$

The linear part with respect to $\tilde{\boldsymbol{\eta}}$ in the difference $\frac{1}{2}\left(a_{\alpha \beta}(\boldsymbol{\eta})-a_{\alpha \beta}\right)$ is called the linearized change of metric tensor associated with the displacement field $\eta_{i} \boldsymbol{a}^{i}$, the covariant components of which are then given by

$$
\begin{aligned}
\gamma_{\alpha \beta}(\boldsymbol{\eta}) & =\frac{1}{2}\left(\boldsymbol{a}_{\alpha} \cdot \partial_{\beta} \tilde{\boldsymbol{\eta}}+\partial_{\alpha} \tilde{\boldsymbol{\eta}} \cdot \boldsymbol{a}_{\beta}\right) \\
& =\frac{1}{2}\left(\partial_{\beta} \eta_{\alpha}+\partial_{\alpha} \eta_{\beta}\right)-\Gamma_{\alpha \beta}^{\sigma} \eta_{\sigma}-b_{\alpha \beta} \eta_{3}=\gamma_{\beta \alpha}(\boldsymbol{\eta}) .
\end{aligned}
$$

Prepared using sagej.cls 
The linear part with respect to $\tilde{\boldsymbol{\eta}}$ in the difference $\left(b_{\alpha \beta}(\boldsymbol{\eta})-b_{\alpha \beta}\right)$ is called the linearized change of curvature tensor associated with the displacement field $\eta_{i} \boldsymbol{a}^{i}$, the covariant components of which are then given by

$$
\begin{aligned}
\rho_{\alpha \beta}(\boldsymbol{\eta}) & =\left(\partial_{\alpha \beta} \tilde{\boldsymbol{\eta}}-\Gamma_{\alpha \beta}^{\sigma} \partial_{\sigma} \tilde{\boldsymbol{\eta}}\right) \cdot \boldsymbol{a}_{3} \\
& =\partial_{\alpha \beta} \eta_{3}-\Gamma_{\alpha \beta}^{\sigma} \partial_{\sigma} \eta_{3}-b_{\alpha}^{\sigma} b_{\sigma \beta} \eta_{3} \\
& +b_{\alpha}^{\sigma}\left(\partial_{\beta} \eta_{\sigma}-\Gamma_{\beta \sigma}^{\tau} \eta_{\tau}\right)+b_{\beta}^{\tau}\left(\partial_{\alpha} \eta_{\tau}-\Gamma_{\alpha \tau}^{\sigma} \eta_{\sigma}\right) \\
& +\left(\partial_{\alpha} b_{\beta}^{\tau}+\Gamma_{\alpha \sigma}^{\tau} b_{\beta}^{\sigma}-\Gamma_{\alpha \beta}^{\sigma} b_{\sigma}^{\tau}\right) \eta_{\tau}=\rho_{\beta \alpha}(\boldsymbol{\eta}) .
\end{aligned}
$$

Let us now define the time-dependent version of the linearized change of metric tensor $\gamma_{\alpha \beta}$. Consider the operator

$$
\tilde{\gamma}_{\alpha \beta}: L^{2}\left(0, T ; H^{1}(\omega) \times H^{1}(\omega) \times L^{2}(\omega)\right) \rightarrow L^{2}\left(0, T ; L^{2}(\omega)\right),
$$

defined by

$$
\tilde{\gamma}_{\alpha \beta}(\boldsymbol{\eta})(t):=\gamma_{\alpha \beta}(\boldsymbol{\eta}(t)) \text { for all } \boldsymbol{\eta} \in L^{2}\left(0, T ; H^{1}(\omega) \times H^{1}(\omega) \times L^{2}(\omega)\right),
$$

for almost all (a.a. in what follows) $t \in(0, T)$.

Let us show that the definition is well-posed, i.e., that for each $\boldsymbol{\eta}$ in $L^{2}\left(0, T ; H^{1}(\omega) \times H^{1}(\omega) \times L^{2}(\omega)\right)$ the following integral

$$
\int_{0}^{T}\left\|\tilde{\gamma}_{\alpha \beta}(\boldsymbol{\eta})(t)\right\|_{0, \omega}^{2} \mathrm{~d} t
$$

is finite.

Clearly, $\gamma_{\alpha \beta}(\boldsymbol{\eta}(t))$ is in $L^{2}(\omega)$, for a.a. $t \in(0, T)$. Observe also that

$$
\int_{0}^{T}\left\|\tilde{\gamma}_{\alpha \beta}(\boldsymbol{\eta})(t)\right\|_{0, \omega}^{2} \mathrm{~d} t=\int_{0}^{T}\left\|\gamma_{\alpha \beta}(\boldsymbol{\eta}(t))\right\|_{0, \omega}^{2} \mathrm{~d} t \leq C \int_{0}^{T}\|\boldsymbol{\eta}(t)\|_{H^{1}(\omega) \times H^{1}(\omega) \times L^{2}(\omega)}^{2} \mathrm{~d} t,
$$

where the constant $C$ is uniform with respect to $t$, since it depends only on the Christoffel symbols and the second fundamental form of the surface $\boldsymbol{\theta}(\bar{\omega})$.

The operator $\tilde{\gamma}_{\alpha \beta}$ is clearly linear. Indeed, for each $\boldsymbol{\xi}, \boldsymbol{\eta}$ in $L^{2}\left(0, T ; H^{1}(\omega) \times\right.$ $\left.H^{1}(\omega) \times L^{2}(\omega)\right)$, we have that

$$
\tilde{\gamma}_{\alpha \beta}(\boldsymbol{\xi}+\boldsymbol{\eta})(t)=\gamma_{\alpha \beta}(\boldsymbol{\xi}(t))+\gamma_{\alpha \beta}(\boldsymbol{\eta}(t))=\left(\tilde{\gamma}_{\alpha \beta}(\boldsymbol{\xi})+\tilde{\gamma}_{\alpha \beta}(\boldsymbol{\eta})\right)(t),
$$

for a.a. $t \in(0, T)$. The fact that $\tilde{\gamma}_{\alpha \beta}(c \boldsymbol{\eta})=c \tilde{\gamma}_{\alpha \beta}(\boldsymbol{\eta})$, for all $c \in \mathbb{R}$ and all $\boldsymbol{\eta} \in L^{2}\left(0, T ; H^{1}(\omega) \times H^{1}(\omega) \times L^{2}(\omega)\right)$, is straightforward.

The operator $\tilde{\gamma}_{\alpha \beta}$ is continuous. Indeed, for each $\boldsymbol{\eta}$ in $L^{2}\left(0, T ; H^{1}(\omega) \times\right.$ $\left.H^{1}(\omega) \times L^{2}(\omega)\right)$, we have that

$$
\begin{aligned}
& \left\|\tilde{\gamma}_{\alpha \beta}(\boldsymbol{\eta})\right\|_{L^{2}\left(0, T ; L^{2}(\omega)\right)}^{2}=\int_{0}^{T}\left\|\gamma_{\alpha \beta}(\boldsymbol{\eta}(t))\right\|_{0, \omega}^{2} \mathrm{~d} t \\
& \leq C\|\boldsymbol{\eta}\|_{L^{2}\left(0, T ; H^{1}(\omega) \times H^{1}(\omega) \times L^{2}(\omega)\right)},
\end{aligned}
$$

Prepared using sagej.cls 
where, again, the constant $C>0$ is uniform with respect to $t$.

The terminology "analogue" for the linear and continuous operator $\tilde{\gamma}_{\alpha \beta}$ is justified by the fact that

$$
\tilde{\gamma}_{\alpha \beta}(\boldsymbol{\eta})=\gamma_{\alpha \beta}(\boldsymbol{\eta}) \text { for all } \boldsymbol{\eta} \in H^{1}(\omega) \times H^{1}(\omega) \times L^{2}(\omega) \text { independent of } t \text {. }
$$

We can similarly define the time-dependent version of the linearized change of curvature tensor $\rho_{\alpha \beta}$. Consider the operator

$$
\tilde{\rho}_{\alpha \beta}: L^{2}\left(0, T ; H^{1}(\omega) \times H^{1}(\omega) \times H^{2}(\omega)\right) \rightarrow L^{2}\left(0, T ; L^{2}(\omega)\right),
$$

defined by

$$
\tilde{\rho}_{\alpha \beta}(\boldsymbol{\eta})(t):=\rho_{\alpha \beta}(\boldsymbol{\eta}(t)) \text { for all } \boldsymbol{\eta} \in L^{2}\left(0, T ; H^{1}(\omega) \times H^{1}(\omega) \times H^{2}(\omega)\right) \text {, }
$$

for a.a. $t \in(0, T)$. This operator is clearly well-defined, linear, and continuous. Moreover, for all $\boldsymbol{\eta} \in H^{1}(\omega) \times H^{1}(\omega) \times H^{2}(\omega)$ independent of $t$, we have

$$
\tilde{\rho}_{\alpha \beta}(\boldsymbol{\eta})=\rho_{\alpha \beta}(\boldsymbol{\eta}) .
$$

\section{A natural model for time-dependent flexural shells}

Let $\omega$ be a domain in $\mathbb{R}^{2}$ with boundary $\gamma$, and let $\gamma_{0}$ be a non-empty relatively open subset of $\gamma$. Let $I$ be an interval of the form $(0, T)$, with $T<\infty$.

For each $\varepsilon>0$, we define the sets

$$
\left.\Omega^{\varepsilon}:=\omega \times\right]-\varepsilon, \varepsilon\left[\text { and } \Gamma_{ \pm}^{\varepsilon}:=\omega \times\{ \pm \varepsilon\},\right.
$$

we let $x^{\varepsilon}=\left(x_{i}^{\varepsilon}\right)$ designate a generic point in the set $\overline{\Omega^{\varepsilon}}$, and let $\partial_{i}^{\varepsilon}:=\partial / \partial x_{i}^{\varepsilon}$. Hence we have $x_{\alpha}^{\varepsilon}=y_{\alpha}$ and $\partial_{\alpha}^{\varepsilon}=\partial_{\alpha}$. Define, also, the set

$$
\Gamma_{0}^{\varepsilon}:=\gamma_{0} \times[-\varepsilon, \varepsilon],
$$

which is thus a subset of the lateral face of the undeformed reference configuration.

In all that follows, we are given an injective immersion $\boldsymbol{\theta} \in \mathcal{C}^{3}\left(\bar{\omega} ; \mathbb{E}^{3}\right)$ and $\varepsilon>0$, and we consider a shell with middle surface $\boldsymbol{\theta}(\bar{\omega})$ and with constant thickness $2 \varepsilon$. This means that the reference configuration of the shell is the set $\boldsymbol{\Theta}\left(\overline{\Omega^{\varepsilon}}\right)$, where the mapping $\boldsymbol{\Theta}: \overline{\Omega^{\varepsilon}} \rightarrow \mathbb{E}^{3}$ is defined by

$$
\boldsymbol{\Theta}\left(x^{\varepsilon}\right):=\boldsymbol{\theta}(y)+x_{3}^{\varepsilon} \boldsymbol{a}^{3}(y) \text { at each point } x^{\varepsilon}=\left(y, x_{3}^{\varepsilon}\right) \in \overline{\Omega^{\varepsilon}} .
$$

Note that the injectivity assumption is made here for physical reasons, but that is otherwise not needed in the proofs. One can then show (cf. Theorem 3.11 of [21] or Theorem 4.1-1 of [22]) that, if the thickness $\varepsilon>0$ is small enough, such a mapping $\Theta \in \mathcal{C}^{2}\left(\overline{\Omega^{\varepsilon}} ; \mathbb{E}^{3}\right)$ is a $\mathcal{C}^{2}$-diffeomorphism from $\overline{\Omega^{\varepsilon}}$ onto $\Theta\left(\overline{\Omega^{\varepsilon}}\right)$, hence is in particular an injective immersion, in the sense that the three vectors

$$
\boldsymbol{g}_{i}^{\varepsilon}\left(x^{\varepsilon}\right):=\partial_{i}^{\varepsilon} \boldsymbol{\Theta}\left(x^{\varepsilon}\right)
$$

Prepared using sagej.cls 
are linearly independent at each point $x^{\varepsilon} \in \overline{\Omega^{\varepsilon}}$; these vectors then constitute the covariant basis at the point $\boldsymbol{\Theta}\left(x^{\varepsilon}\right)$, while the three vectors $\boldsymbol{g}^{j, \varepsilon}\left(x^{\varepsilon}\right)$, defined by the relations

$$
\boldsymbol{g}^{j, \varepsilon}\left(x^{\varepsilon}\right) \cdot \boldsymbol{g}_{i}^{\varepsilon}\left(x^{\varepsilon}\right)=\delta_{i}^{j},
$$

constitute the contravariant basis at the same point.

It will be implicitly assumed in what follows that the immersion $\boldsymbol{\theta} \in$ $\mathcal{C}^{3}\left(\bar{\omega} ; \mathbb{E}^{3}\right)$ is injective and that $\varepsilon>0$ is small enough so that $\Theta: \overline{\Omega^{\varepsilon}} \rightarrow \mathbb{E}^{3}$ is $a \mathcal{C}^{2}$-diffeomorphism onto its image.

We henceforth assume that the shell is made of a homogeneous and isotropic linearly elastic material and that its reference configuration $\Theta\left(\overline{\Omega^{\varepsilon}}\right)$ is a natural state, i.e., is stress free. As a result of these assumptions, the elastic behavior of this elastic material is completely characterized by its two Lamé constants $\lambda \geq 0$ and $\mu>0$ (see, e.g., Section 3.8 in [24]). The positive constant $\rho$ designates the mass density of the shell per unit volume.

We also assume that the shell is subjected to applied body forces whose density per unit volume is defined by means of its contravariant components $f^{i, \varepsilon} \in L^{\infty}\left(0, T ; L^{2}\left(\Omega^{\varepsilon}\right)\right)$, i.e., over the vectors $\boldsymbol{g}_{i}^{\varepsilon}$ of the covariant bases; to applied surface forces whose density per unit area is defined by means of its contravariant components $h^{i, \varepsilon} \in L^{\infty}\left(0, T ; L^{2}\left(\Gamma_{+}^{\varepsilon} \cup \Gamma_{-}^{\varepsilon}\right)\right)$, i.e., over the vectors $\boldsymbol{g}_{i}^{\varepsilon}$ of the covariant bases; and to a homogeneous boundary condition of place along the portion $\Gamma_{0}^{\varepsilon}$ of its lateral face, i.e., the admissible displacement fields vanish on $\Gamma_{0}^{\varepsilon}$. For a.a. $t \in(0, T)$, we can thus define the contravariant components $p^{i, \varepsilon}(t)$ of the vector $\boldsymbol{p}^{\varepsilon}=\left(p^{i, \varepsilon}\right)$ over the vectors $\boldsymbol{a}_{i}$ of the covariant bases by

$$
p^{i, \varepsilon}(t):=\left\{\int_{-\varepsilon}^{\varepsilon} f^{i, \varepsilon}(t) \mathrm{d} x_{3}^{\varepsilon}+h_{+}^{i, \varepsilon}(t)+h_{-}^{i, \varepsilon}(t)\right\} \in L^{2}(\omega) \text { for a.a. } t \in(0, T),
$$

where $h_{ \pm}^{i, \varepsilon}(t):=h^{i, \varepsilon}(t)(\cdot, \pm \varepsilon) \in L^{2}(\omega)$, for a.a. $t \in(0, T)$.

Define the space

$$
\boldsymbol{V}_{K}(\omega):=\left\{\boldsymbol{\eta}=\left(\eta_{i}\right) \in H^{1}(\omega) \times H^{1}(\omega) \times H^{2}(\omega) ; \eta_{i}=\partial_{\nu} \eta_{3}=0 \text { on } \gamma_{0}\right\}
$$

where the symbol $\partial_{\nu}$ denotes the outer unit normal derivative operator along $\gamma$. The space $\boldsymbol{V}_{K}(\omega)$ is the one used for formulating the two-dimensional equations governing Koiter's model (see the series of papers [25], [11], [18] and [17]).

Define the norm $\|\cdot\|_{\boldsymbol{V}_{K}(\omega)}$ by

$$
\|\boldsymbol{\eta}\|_{\boldsymbol{V}_{K}(\omega)}:=\left\{\sum_{\alpha}\left\|\eta_{\alpha}\right\|_{1, \omega}^{2}+\left\|\eta_{3}\right\|_{2, \omega}^{2}\right\}^{1 / 2} \quad \text { for each } \boldsymbol{\eta}=\left(\eta_{i}\right) \in \boldsymbol{V}_{K}(\omega),
$$

Next, we define the fourth-order two-dimensional elasticity tensor of the shell, viewed here as a two-dimensional linearly elastic body, by means of its contravariant components

$$
a^{\alpha \beta \sigma \tau}:=\frac{4 \lambda \mu}{\lambda+2 \mu} a^{\alpha \beta} a^{\sigma \tau}+2 \mu\left(a^{\alpha \sigma} a^{\beta \tau}+a^{\alpha \tau} a^{\beta \sigma}\right) .
$$

Prepared using sagej.cls 
Following the terminology proposed in Section 6.1 of [21], a linearly elastic shell is said to be a flexural shell if the following two additional assumptions are satisfied: first, length $\gamma_{0}>0$ (an assumption that is satisfied if $\gamma_{0}$ is a nonempty relatively open subset of $\gamma$, as here), and second, the following space of admissible linearized inextensional displacements:

$$
\begin{array}{r}
\boldsymbol{V}_{F}(\omega):=\left\{\boldsymbol{\eta}=\left(\eta_{i}\right) \in H^{1}(\omega) \times H^{1}(\omega) \times H^{2}(\omega) ;\right. \\
\left.\eta_{i}=\partial_{\nu} \eta_{3}=0 \text { on } \gamma_{0} \text { and } \gamma_{\alpha \beta}(\boldsymbol{\eta})=0 \text { in } \omega\right\},
\end{array}
$$

contains nonzero functions, i.e.,

$$
\boldsymbol{V}_{F}(\omega) \neq\{\mathbf{0}\} .
$$

Classical examples of flexural shells are, for instance, cylindrical shells, conical shells and plates (see, respectively, Figures 6.1-1, 6.1-2, and 6.1-3 of [21]).

To begin with, we state a crucial inequality that holds for general surfaces.

Theorem 3.1. Let $\omega$ be a domain in $\mathbb{R}^{2}$ and let $\boldsymbol{\theta} \in \mathcal{C}^{3}\left(\bar{\omega} ; \mathbb{E}^{3}\right)$ be an immersion. Let $\gamma_{0}$ be a non-empty relatively open subset of $\gamma$. Define the space

$$
\boldsymbol{V}_{K}(\omega):=\left\{\boldsymbol{\eta}=\left(\eta_{i}\right) \in H^{1}(\omega) \times H^{1}(\omega) \times H^{2}(\omega) ; \eta_{i}=\partial_{\nu} \eta_{3}=0 \text { on } \gamma_{0}\right\} .
$$

Then there exists a constant $c=c\left(\omega, \gamma_{0}, \boldsymbol{\theta}\right)>0$ such that

$$
\left\{\sum_{\alpha}\left\|\eta_{\alpha}\right\|_{1, \omega}^{2}+\left\|\eta_{3}\right\|_{2, \omega}^{2}\right\}^{1 / 2} \leq c\left\{\sum_{\alpha, \beta}\left\|\gamma_{\alpha \beta}(\boldsymbol{\eta})\right\|_{0, \omega}^{2}+\sum_{\alpha, \beta}\left\|\rho_{\alpha \beta}(\boldsymbol{\eta})\right\|_{0, \omega}^{2}\right\}^{1 / 2}
$$

for all $\boldsymbol{\eta}=\left(\eta_{i}\right) \in \boldsymbol{V}_{K}(\omega)$.

The above inequality, which is due to Bernadou \& Ciarlet [26] and was later improved by Bernadou, Ciarlet \& Miara [27] (see also Theorem 2.6-4 of [21]), constitutes an example of a Korn inequality on a general surface; it constitutes a "Korn inequality" in the sense that it provides a basic estimate of an appropriate norm of a displacement field defined on a surface in terms of an appropriate norm of a specific "measure of strain" (here, the linearized change of metric tensor and the linearized change of curvature tensor) corresponding to the displacement field under consideration.

A natural formulation of a set of time-dependent two-dimensional equations ("two-dimensional", in the sense that they are posed over the two-dimensional subset $\omega$ ) can be derived by slightly modifying the model proposed by Xiao in the paper [20], where time-dependent Koiter's shells are studied.

Let us introduce the problem $\mathcal{P}_{F}^{\varepsilon}(\omega)$, which constitutes the point of departure of our analysis.

Problem $\mathcal{P}_{F}^{\varepsilon}(\omega)$. Find a vector field $\boldsymbol{\zeta}^{\varepsilon}=\left(\zeta_{i}^{\varepsilon}\right):(0, T) \rightarrow \boldsymbol{V}_{F}(\omega)$ such that

$$
\begin{aligned}
& \boldsymbol{\zeta}^{\varepsilon} \in L^{\infty}\left(0, T ; \boldsymbol{V}_{F}(\omega)\right), \\
& \dot{\boldsymbol{\zeta}}^{\varepsilon} \in L^{\infty}\left(0, T ; \boldsymbol{L}^{2}(\omega)\right), \\
& \ddot{\boldsymbol{\zeta}}^{\varepsilon} \in L^{\infty}\left(0, T ; \boldsymbol{V}_{F}^{*}(\omega)\right),
\end{aligned}
$$

Prepared using sagej.cls 
that satisfies the following variational equations

$2 \varepsilon^{3} \rho \frac{\mathrm{d}^{2}}{\mathrm{~d} t^{2}} \int_{\omega} \zeta_{i}^{\varepsilon}(t) \eta_{i} \sqrt{a} \mathrm{~d} y+\frac{\varepsilon^{3}}{3} \int_{\omega} a^{\alpha \beta \sigma \tau} \rho_{\sigma \tau}\left(\boldsymbol{\zeta}^{\varepsilon}(t)\right) \rho_{\alpha \beta}(\boldsymbol{\eta}) \sqrt{a} \mathrm{~d} y=\int_{\omega} p^{i, \varepsilon}(t) \eta_{i} \sqrt{a} \mathrm{~d} y$,

for all $\boldsymbol{\eta}=\left(\eta_{i}\right) \in \boldsymbol{V}_{F}(\omega)$, in the sense of distributions in $(0, T)$, and that satisfies the following initial conditions

$$
\left\{\begin{array}{l}
\zeta^{\varepsilon}(0)=\zeta_{0} \\
\dot{\zeta}^{\varepsilon}(0)=\zeta_{1}
\end{array}\right.
$$

where $\boldsymbol{\zeta}_{0} \in \boldsymbol{V}_{F}(\omega)$ and $\boldsymbol{\zeta}_{1} \in \boldsymbol{L}^{2}(\omega)$ are prescribed.

We say that $\boldsymbol{\zeta}^{\varepsilon}$ is a weak solution of Problem $\mathcal{P}_{F}^{\varepsilon}(\omega)$ if

$$
\begin{aligned}
& \boldsymbol{\zeta}^{\varepsilon} \in L^{\infty}\left(0, T ; \boldsymbol{V}_{F}(\omega)\right), \\
& \dot{\boldsymbol{\zeta}}^{\varepsilon} \in L^{\infty}\left(0, T ; \boldsymbol{L}^{2}(\omega)\right), \\
& \ddot{\boldsymbol{\zeta}}^{\varepsilon} \in L^{\infty}\left(0, T ; \boldsymbol{V}_{F}^{*}(\omega)\right),
\end{aligned}
$$

if $\zeta^{\varepsilon}$ satisfies the variational equations of Problem $\mathcal{P}_{F}^{\varepsilon}(\omega)$ in the sense of distributions in $(0, T)$, and also satisfies the initial conditions (1).

We say that $\boldsymbol{\zeta}^{\varepsilon}$ is a strong solution of Problem $\mathcal{P}_{F}^{\varepsilon}(\omega)$ if

$$
\boldsymbol{\zeta}^{\varepsilon} \in \mathcal{C}^{0}\left([0, T] ; \boldsymbol{V}_{F}(\omega)\right) \cap \mathcal{C}^{1}\left([0, T] ; \boldsymbol{L}^{2}(\omega)\right),
$$

if $\boldsymbol{\zeta}^{\varepsilon}$ satisfies the variational equations of Problem $\mathcal{P}_{F}^{\varepsilon}(\omega)$ in the sense of distributions in $(0, T)$, and also satisfies the initial conditions (1).

We recall a very important inequality which is used to study evolutionary problems: Gronwall's inequality (see the seminal paper [28] and Theorem 1.1 in Chapter III of [29]).

Theorem 3.2. Let $T>0$ and suppose that the function $y:[0, T] \rightarrow \mathbb{R}$ is absolutely continuous and such that

$$
\frac{\mathrm{d} y}{\mathrm{~d} t}(t) \leq a(t) y(t)+b(t), \quad \text { for a.a. } t \in(0, T),
$$

where $a, b \in L^{1}(0, T)$ and $a, b \geq 0$ for a.a. $t \in(0, T)$. Then, it results

$$
y(t) \leq\left[y(0)+\int_{0}^{t} b(s) \mathrm{d} s\right] e^{\int_{0}^{t} a(s) \mathrm{d} s}, \quad \text { for all } t \in[0, T] .
$$

\section{Existence and uniqueness of solutions of Problem $\mathcal{P}_{F}^{\varepsilon}(\omega)$ : Classical approach}

The proof of existence and uniqueness of strong solutions of Problem $\mathcal{P}_{F}^{\varepsilon}(\omega)$ can be straightforwardly obtained by implementing the same strategy as in Section 6 of Chapter 1 of [30]. In all what follows, the symbol “ $\hookrightarrow$ " denotes a continuous embedding, while the symbol " $\hookrightarrow$ " denotes a compact embedding.

Prepared using sagej.cls 
Theorem 4.1. Problem $\mathcal{P}_{F}^{\varepsilon}(\omega)$ admits a unique strong solution $\boldsymbol{\zeta}^{\varepsilon} \in$ $\mathcal{C}^{0}\left([0, T] ; \boldsymbol{V}_{F}(\omega)\right) \cap \mathcal{C}^{1}\left([0, T] ; \boldsymbol{L}^{2}(\omega)\right)$.

Proof. Define

$$
\boldsymbol{H}_{F}(\omega):={\overline{\boldsymbol{V}_{F}(\omega)}}^{\|\cdot\|_{L^{2}(\omega)}}
$$

and let us observe that it is a closed subspace of $\boldsymbol{L}^{2}(\omega)$. Indeed, given any $\boldsymbol{\eta}$, $\boldsymbol{\xi} \in \boldsymbol{H}_{F}(\omega)$, there exist sequences $\left(\boldsymbol{\eta}_{k}\right)_{k=1}^{\infty}$ and $\left(\boldsymbol{\xi}_{k}\right)_{k=1}^{\infty}$, both of them in $\boldsymbol{V}_{F}(\omega)$, such that

$$
\begin{aligned}
& \boldsymbol{\eta}_{k} \rightarrow \boldsymbol{\eta}, \quad \text { in } \boldsymbol{L}^{2}(\omega) \text { as } k \rightarrow \infty, \\
& \boldsymbol{\xi}_{k} \rightarrow \boldsymbol{\xi}, \quad \text { in } \boldsymbol{L}^{2}(\omega) \text { as } k \rightarrow \infty .
\end{aligned}
$$

Let us now consider the sequence $\left(\boldsymbol{\Upsilon}_{k}\right)_{k=1}^{\infty}$, where

$$
\boldsymbol{\Upsilon}_{k}:=\boldsymbol{\eta}_{k}+\boldsymbol{\xi}_{k}, \quad \text { for each } k \geq 1,
$$

and observe that $\left(\boldsymbol{\Upsilon}_{k}\right)_{k=1}^{\infty}$ is contained in $\boldsymbol{V}_{F}(\omega)$, since the constraints appearing in the definition of the space $\boldsymbol{V}_{F}(\omega)$ are linear. Hence, we have

$$
\Upsilon_{k} \rightarrow(\boldsymbol{\eta}+\boldsymbol{\xi}), \quad \text { in } \boldsymbol{L}^{2}(\omega) \text { as } k \rightarrow \infty,
$$

which shows that the element $(\boldsymbol{\eta}+\boldsymbol{\xi})$ belongs to $\boldsymbol{H}_{F}(\omega)$. Likewise, it can be shown that, given any $\alpha \in \mathbb{R}$ and any $\boldsymbol{\eta} \in \boldsymbol{H}_{F}(\omega)$, it results $(\alpha \boldsymbol{\eta}) \in \boldsymbol{H}_{F}(\omega)$.

It can also be observed that the following chain of embeddings holds if we identify $\boldsymbol{H}_{F}(\omega)$ with its dual (see, e.g., Lemma 6.8 on page 74 of [30])

$$
\boldsymbol{V}_{F}(\omega) \hookrightarrow \hookrightarrow \boldsymbol{H}_{F}(\omega) \hookrightarrow \hookrightarrow \boldsymbol{V}_{F}^{*}(\omega) .
$$

Since $\boldsymbol{V}_{F}(\omega)$ is clearly dense in $\boldsymbol{H}_{F}(\omega)$ with respect to the norm $\|\cdot\|_{\boldsymbol{L}^{2}(\omega)}$, we are in a position to apply Theorem 8.2-2 of [31] and infer the existence and uniqueness of strong solutions of Problem $\mathcal{P}_{F}^{\varepsilon}(\omega)$. This completes the proof.

The proof presented above is straightforward and resorts to classical results. However, in the context of the implementation of numerical schemes, the involved function spaces are not amenable for the construction of a finite element basis. We recall, indeed, that it is often very complicated to construct a finite element basis within a function space bearing a constraint.

\section{Penalty scheme for the considered problem}

To fix the ideas, from now onward, we identify $L^{2}(\omega)$ and $\boldsymbol{L}^{2}(\omega)$ with their respective dual spaces, and we equip them with the following inner products

$$
\begin{aligned}
(\eta, \xi) & \in L^{2}(\omega) \times L^{2}(\omega) \rightarrow \int_{\omega} \eta \xi \sqrt{a} \mathrm{~d} y, \\
(\boldsymbol{\eta}, \boldsymbol{\xi}) \in \boldsymbol{L}^{2}(\omega) \times \boldsymbol{L}^{2}(\omega) & \rightarrow \int_{\omega} \eta_{i} \xi_{i} \sqrt{a} \mathrm{~d} y .
\end{aligned}
$$

Prepared using sagej.cls 
A possible way to prove the existence and uniqueness of solutions of Problem $\mathcal{P}_{F}^{\varepsilon}(\omega)$ without relying on the abstract functional spaces introduced in Theorem 4.1, consists in adapting the penalty scheme described in Chapter II, Section 4 of [32] (see also [33]) to formulate an alternate problem posed over the function space $\boldsymbol{V}_{K}(\omega)$, which does not take into account the constraint appearing in the definition of the space $\boldsymbol{V}_{F}(\omega)$.

Observe first that $\boldsymbol{V}_{K}(\omega)$ is dense in $\boldsymbol{L}^{2}(\omega)$ and that

$$
\boldsymbol{V}_{K}(\omega) \hookrightarrow \hookrightarrow \boldsymbol{L}^{2}(\omega) \hookrightarrow \hookrightarrow \boldsymbol{V}_{K}^{*}(\omega)
$$

Let $\kappa>0$ denote the penalty parameter and let us introduce the corresponding "penalized" problem $\mathcal{P}_{F, \kappa}^{\varepsilon}(\omega)$.

Problem $\mathcal{P}_{F, \kappa}^{\varepsilon}(\omega)$. Find a vector field $\boldsymbol{\zeta}_{\kappa}^{\varepsilon}=\left(\zeta_{i, \kappa}^{\varepsilon}\right):[0, T] \rightarrow \boldsymbol{V}_{K}(\omega)$ such that

$$
\boldsymbol{\zeta}_{\kappa}^{\varepsilon} \in \mathcal{C}^{0}\left([0, T] ; \boldsymbol{V}_{K}(\omega)\right) \cap \mathcal{C}^{1}\left([0, T] ; \boldsymbol{L}^{2}(\omega)\right)
$$

that satisfies the following variational equations

$$
\begin{gathered}
2 \varepsilon^{3} \rho \frac{\mathrm{d}^{2}}{\mathrm{~d} t^{2}} \int_{\omega} \zeta_{i, \kappa}^{\varepsilon}(t) \eta_{i} \sqrt{a} \mathrm{~d} y+\frac{\varepsilon^{3}}{3} \int_{\omega} a^{\alpha \beta \sigma \tau} \rho_{\sigma \tau}\left(\boldsymbol{\zeta}_{\kappa}^{\varepsilon}(t)\right) \rho_{\alpha \beta}(\boldsymbol{\eta}) \sqrt{a} \mathrm{~d} y \\
+\frac{1}{\kappa} \int_{\omega} a^{\alpha \beta \sigma \tau} \gamma_{\sigma \tau}\left(\boldsymbol{\zeta}_{\kappa}^{\varepsilon}(t)\right) \gamma_{\alpha \beta}(\boldsymbol{\eta}) \sqrt{a} \mathrm{~d} y=\int_{\omega} p^{i, \varepsilon}(t) \eta_{i} \sqrt{a} \mathrm{~d} y
\end{gathered}
$$

for all $\boldsymbol{\eta} \in \boldsymbol{V}_{K}(\omega)$, in the sense of distributions in $(0, T)$, and which satisfies the initial conditions (1).

We say that $\boldsymbol{\zeta}_{\kappa}^{\varepsilon}$ is a weak solution of Problem $\mathcal{P}_{F, \kappa}^{\varepsilon}(\omega)$ if

$$
\begin{aligned}
& \boldsymbol{\zeta}_{\kappa}^{\varepsilon} \in L^{\infty}\left(0, T ; \boldsymbol{V}_{K}(\omega)\right), \\
& \dot{\boldsymbol{\zeta}}_{\kappa}^{\varepsilon} \in L^{\infty}\left(0, T ; \boldsymbol{L}^{2}(\omega)\right), \\
& \ddot{\boldsymbol{\zeta}}_{\kappa}^{\varepsilon} \in L^{\infty}\left(0, T ; \boldsymbol{V}_{K}^{*}(\omega)\right),
\end{aligned}
$$

if $\boldsymbol{\zeta}_{\kappa}^{\varepsilon}$ satisfies the variational equations of Problem $\mathcal{P}_{F, \kappa}^{\varepsilon}(\omega)$ in the sense of distributions in $(0, T)$, and also satisfies the initial conditions (1).

We say that $\boldsymbol{\zeta}_{\kappa}^{\varepsilon}$ is a strong solution of Problem $\mathcal{P}_{F, \kappa}^{\varepsilon}(\omega)$ if

$$
\boldsymbol{\zeta}_{\kappa}^{\varepsilon} \in \mathcal{C}^{0}\left([0, T] ; \boldsymbol{V}_{K}(\omega)\right) \cap \mathcal{C}^{1}\left([0, T] ; \boldsymbol{L}^{2}(\omega)\right)
$$

if $\boldsymbol{\zeta}_{\kappa}^{\varepsilon}$ satisfies the variational equations of Problem $\mathcal{P}_{F, \kappa}^{\varepsilon}(\omega)$ in the sense of distributions in $(0, T)$, and also satisfies the initial conditions (1).

For each $\kappa>0$, let us define the bilinear form $a_{\kappa}: V_{K}(\omega) \times \boldsymbol{V}_{K}(\omega) \rightarrow \mathbb{R}$ by

$$
a_{\kappa}(\boldsymbol{\xi}, \boldsymbol{\eta}):=\frac{\varepsilon^{3}}{3} \int_{\omega} a^{\alpha \beta \sigma \tau} \rho_{\sigma \tau}(\boldsymbol{\xi}) \rho_{\alpha \beta}(\boldsymbol{\eta}) \sqrt{a} \mathrm{~d} y+\frac{1}{\kappa} \int_{\omega} a^{\alpha \beta \sigma \tau} \gamma_{\sigma \tau}(\boldsymbol{\xi}) \gamma_{\alpha \beta}(\boldsymbol{\eta}) \sqrt{a} \mathrm{~d} y
$$

Prepared using sagej.cls 
The bilinear form $a_{\kappa}(\cdot, \cdot)$ is continuous over the space $\boldsymbol{V}_{K}(\omega)$, i.e., there exists a constant $C_{\kappa}>0$, which depends on $\kappa$, such that

$$
\left|a_{\kappa}(\boldsymbol{\xi}, \boldsymbol{\eta})\right| \leq C_{\kappa}\|\boldsymbol{\xi}\|_{\boldsymbol{V}_{K}(\omega)}\|\boldsymbol{\eta}\|_{\boldsymbol{V}_{K}(\omega)}, \quad \text { for all } \boldsymbol{\xi}, \boldsymbol{\eta} \in \boldsymbol{V}_{K}(\omega)
$$

For $\kappa>0$ sufficiently small (recall that the small parameter $\varepsilon>0$ is fixed), the uniform positive-definiteness of the elasticity tensor of the shell $\left(a^{\alpha \beta \sigma \tau}\right)$ (cf. Theorem 3.3-2 of [21]) and Korn inequality on a general surface (Theorem 3.1) give the existence of a constant $c>0$ such that

$$
\begin{aligned}
a_{\kappa}(\boldsymbol{\eta}, \boldsymbol{\eta}) & =\frac{\varepsilon^{3}}{3} \int_{\omega} a^{\alpha \beta \sigma \tau} \rho_{\sigma \tau}(\boldsymbol{\eta}) \rho_{\alpha \beta}(\boldsymbol{\eta}) \sqrt{a} \mathrm{~d} y+\frac{1}{\kappa} \int_{\omega} a^{\alpha \beta \sigma \tau} \gamma_{\sigma \tau}(\boldsymbol{\eta}) \gamma_{\alpha \beta}(\boldsymbol{\eta}) \sqrt{a} \mathrm{~d} y \\
& \geq \frac{\varepsilon^{3}}{3} \sum_{\alpha, \beta}\left\{\left\|\rho_{\alpha \beta}(\boldsymbol{\eta})\right\|_{0, \omega}^{2}+\left\|\gamma_{\alpha \beta}(\boldsymbol{\eta})\right\|_{0, \omega}^{2}\right\} \\
& \geq c\left\{\sum_{\alpha}\left\|\eta_{\alpha}\right\|_{1, \omega}^{2}+\left\|\eta_{3}\right\|_{2, \omega}^{2}\right\}
\end{aligned}
$$

for all $\boldsymbol{\eta} \in \boldsymbol{V}_{K}(\omega)$, namely, the bilinear form $a_{\kappa}(\cdot, \cdot)$ is $\boldsymbol{V}_{K}(\omega)$-elliptic.

We first prove, by Galerkin method, that Problem $\mathcal{P}_{F, \kappa}^{\varepsilon}(\omega)$ admits a unique strong solution.

Theorem 5.1. Problem $\mathcal{P}_{F, \kappa}^{\varepsilon}(\omega)$ admits a unique strong solution $\boldsymbol{\zeta}_{\kappa}^{\varepsilon} \in$ $\mathcal{C}^{0}\left([0, T] ; \boldsymbol{V}_{K}(\omega)\right) \cap \mathcal{C}^{1}\left([0, T] ; \boldsymbol{L}^{2}(\omega)\right)$.

Proof. (i)Construction of Galerkin approximation. Observe that the space $\boldsymbol{V}_{K}(\omega)$ is an infinite-dimensional and separable Hilbert space. Therefore, by Theorem $6.2-1$ of [31], there exists an orthonormal Hilbert basis $\left(\boldsymbol{w}^{k}\right)_{k=1}^{\infty}$ of the space $\boldsymbol{L}^{2}(\omega)$ which also constitutes an orthogonal Hilbert basis of the space $V_{K}(\omega)$.

For each positive integer $m \geq 1$, let us denote by $\boldsymbol{E}^{m}$ the following $m$-dimensional linear hull

$$
\boldsymbol{E}^{m}:=\operatorname{Span}\left(\boldsymbol{w}^{k}\right)_{k=1}^{m} \subset \boldsymbol{V}_{K}(\omega) \subset \boldsymbol{L}^{2}(\omega)
$$

Since each element of the Hilbert basis $\left(\boldsymbol{w}^{k}\right)_{k=1}^{\infty}$ is independent of the time variable $t$, we have $\boldsymbol{w}^{k} \in L^{\infty}\left(0, T ; \boldsymbol{V}_{K}(\omega)\right)$, for each integer $1 \leq k \leq m$. We now discretize Problem $\mathcal{P}_{F, \kappa}^{\varepsilon}(\omega)$ and, in order to keep the notation simple, we drop the dependence of the vector fields entering the variational equations on the parameters $\kappa$ and $\varepsilon$. Let us observe that, the duality pair between $\boldsymbol{E}^{m}$ and its dual coincides with the inner product of $\boldsymbol{L}^{2}(\omega)$ defined in Section 2.

For each positive integer $m \geq 1$, the "penalized" discrete problem corresponding to Problem $\mathcal{P}_{F, \kappa}^{\varepsilon}(\omega)$, that we denote by $\mathcal{P}_{F, \kappa}^{\varepsilon, m}(\omega)$, amounts to:

Problem $\mathcal{P}_{F, \kappa}^{\varepsilon, m}(\omega)$. Find functions $c_{k}:[0, T] \rightarrow \mathbb{R}, 1 \leq k \leq m$, such that

$$
\boldsymbol{\zeta}^{m}(t):=\sum_{k=1}^{m} c_{k}(t) \boldsymbol{w}^{k}, \text { for a.a. } t \in(0, T),
$$

Prepared using sagej.cls 
which satisfies the following variational equations in the sense of distributions in $(0, T)$, for each integer $1 \leq p \leq m$

$$
\begin{aligned}
& 2 \rho \varepsilon^{3} \int_{\omega} \ddot{\zeta}_{i}^{m}(t) w_{i}^{p} \sqrt{a} \mathrm{~d} y \\
& \quad+\frac{\varepsilon^{3}}{3} \int_{\omega} a^{\alpha \beta \sigma \tau} \rho_{\sigma \tau}\left(\boldsymbol{\zeta}^{m}(t)\right) \rho_{\alpha \beta}\left(\boldsymbol{w}^{p}\right) \sqrt{a} \mathrm{~d} y \\
& \quad+\frac{1}{\kappa} \int_{\omega} a^{\alpha \beta \sigma \tau} \gamma_{\sigma \tau}\left(\boldsymbol{\zeta}^{m}(t)\right) \gamma_{\alpha \beta}\left(\boldsymbol{w}^{p}\right) \sqrt{a} \mathrm{~d} y \\
& =\int_{\omega} p^{i, \varepsilon}(t) w_{i}^{p} \sqrt{a} \mathrm{~d} y,
\end{aligned}
$$

and for which the following initial conditions hold:

$$
\left\{\begin{array}{l}
\boldsymbol{\zeta}^{m}(0)=\boldsymbol{\zeta}_{0}^{m}, \\
\dot{\boldsymbol{\zeta}}^{m}(0)=\boldsymbol{\zeta}_{1}^{m},
\end{array}\right.
$$

where initial data $\boldsymbol{\zeta}_{0}^{m}$ and $\boldsymbol{\zeta}_{1}^{m}$ are, respectively, the projections of $\boldsymbol{\zeta}_{0}$ and $\boldsymbol{\zeta}_{1}$ onto the finite dimensional space $\boldsymbol{E}^{m}$.

We immediately observe that the projections of $\boldsymbol{\zeta}_{0}=\left(\zeta_{i, 0}\right)$ and $\boldsymbol{\zeta}_{1}=\left(\zeta_{i, 1}\right)$ onto $\boldsymbol{E}^{m}$ can be expanded as follows (cf. Theorem 4.9-1 of [34])

$$
\begin{aligned}
\boldsymbol{\zeta}_{0}^{m} & =\sum_{k=1}^{m}\left(\int_{\omega} \zeta_{i, 0} w_{i}^{k} \sqrt{a} \mathrm{~d} y+\int_{\omega} \partial_{\alpha} \zeta_{i, 0} \partial_{\alpha} w_{i}^{k} \sqrt{a} \mathrm{~d} y+\int_{\omega} \partial_{\alpha \beta} \zeta_{3,0} \partial_{\alpha \beta} w_{3}^{k} \sqrt{a} \mathrm{~d} y\right) \boldsymbol{w}^{k}, \\
\boldsymbol{\zeta}_{1}^{m} & =\sum_{k=1}^{m}\left(\int_{\omega} \zeta_{i, 1} w_{i}^{k} \sqrt{a} \mathrm{~d} y\right) \boldsymbol{w}^{k}
\end{aligned}
$$

so that $\boldsymbol{\zeta}_{0}^{m} \rightarrow \boldsymbol{\zeta}_{0}$, in $\boldsymbol{V}_{K}(\omega)$ and $\boldsymbol{\zeta}_{1}^{m} \rightarrow \boldsymbol{\zeta}_{1}$, in $\boldsymbol{L}^{2}(\omega)$.

Since the elements of the Hilbert basis do not depend on the time variable we can take the coefficients $c_{k}$ as well as their derivatives $\dot{c}_{k}$ and $\ddot{c}_{k}$ outside the integral sign. This gives, for each $1 \leq k \leq m$, the following second order linear ordinary differential equation with respect to the variable $t$

$$
\begin{aligned}
& 2 \varepsilon^{3} \rho \ddot{c}_{k}(t)+a_{\kappa}\left(\boldsymbol{w}^{k}, \boldsymbol{w}^{k}\right) c_{k}(t)=\int_{\omega} p^{i, \varepsilon}(t) w_{i}^{k} \sqrt{a} \mathrm{~d} y, \\
& c_{k}(0)=\int_{\omega} \zeta_{i, 0} w_{i}^{k} \sqrt{a} \mathrm{~d} y+\int_{\omega} \partial_{\alpha} \zeta_{i, 0} \partial_{\alpha} w_{i}^{k} \sqrt{a} \mathrm{~d} y+\int_{\omega} \partial_{\alpha \beta} \zeta_{3,0} \partial_{\alpha \beta} w_{3}^{k} \sqrt{a} \mathrm{~d} y, \\
& \dot{c}_{k}(0)=\int_{\omega} \zeta_{i, 1} w_{i}^{k} \sqrt{a} \mathrm{~d} y .
\end{aligned}
$$

Such an ordinary differential equation admits a unique solution, which clearly depends on the parameters $\kappa$ and $\varepsilon$.

(ii) Energy estimates. Let us multiply the variational equations in Problem $\mathcal{P}_{F, \kappa}^{\varepsilon, m}(\omega)$ by $\dot{c}_{k}(t)$ and sum with respect to $k$ varying in the

Prepared using sagej.cls 
set $\{1, \ldots, m\}$. As a result, we obtain that the variational equations in Problem $\mathcal{P}_{F, \kappa}^{\varepsilon, m}(\omega)$ take the form

$$
\begin{aligned}
& \rho \varepsilon^{3} \\
& \quad \frac{\mathrm{d}}{\mathrm{d} t} \int_{\omega} \dot{\zeta}_{i}^{m}(t) \dot{\zeta}_{i}^{m}(t) \sqrt{a} \mathrm{~d} y \\
& \quad+\frac{\varepsilon^{3}}{6} \frac{\mathrm{d}}{\mathrm{d} t} \int_{\omega} a^{\alpha \beta \sigma \tau} \rho_{\sigma \tau}\left(\zeta^{m}(t)\right) \rho_{\alpha \beta}\left(\zeta^{m}(t)\right) \sqrt{a} \mathrm{~d} y \\
& \quad+\frac{1}{2 \kappa} \frac{\mathrm{d}}{\mathrm{d} t} \int_{\omega} a^{\alpha \beta \sigma \tau} \gamma_{\sigma \tau}\left(\zeta^{m}(t)\right) \gamma_{\alpha \beta}\left(\zeta^{m}(t)\right) \sqrt{a} \mathrm{~d} y \\
& =\int_{\omega} p^{i, \varepsilon}(t) \dot{\zeta}_{i}^{m}(t) \sqrt{a} \mathrm{~d} y,
\end{aligned}
$$

for a.a. $t \in(0, T)$.

Carrying out an integration over the interval $(0, t)$, where $0<t \leq T$, changes (3) into

$$
\begin{aligned}
& \rho \varepsilon^{3} \int_{\omega} \dot{\zeta}_{i}^{m}(t) \dot{\zeta}_{i}^{m}(t) \sqrt{a} \mathrm{~d} y \\
& \quad+\frac{\varepsilon^{3}}{6} \int_{\omega} a^{\alpha \beta \sigma \tau} \rho_{\sigma \tau}\left(\boldsymbol{\zeta}^{m}(t)\right) \rho_{\alpha \beta}\left(\boldsymbol{\zeta}^{m}(t)\right) \sqrt{a} \mathrm{~d} y \\
& \quad+\frac{1}{2 \kappa} \int_{\omega} a^{\alpha \beta \sigma \tau} \gamma_{\sigma \tau}\left(\boldsymbol{\zeta}^{m}(t)\right) \gamma_{\alpha \beta}\left(\boldsymbol{\zeta}^{m}(t)\right) \sqrt{a} \mathrm{~d} y \\
&=\rho \varepsilon^{3} \int_{\omega} \zeta_{i, 1}^{m} \zeta_{i, 1}^{m} \sqrt{a} \mathrm{~d} y+\frac{\varepsilon^{3}}{6} \int_{\omega} a^{\alpha \beta \sigma \tau} \rho_{\sigma \tau}\left(\boldsymbol{\zeta}^{m}(0)\right) \rho_{\alpha \beta}\left(\boldsymbol{\zeta}^{m}(0)\right) \sqrt{a} \mathrm{~d} y \\
& \quad+\frac{1}{2 \kappa} \int_{\omega} a^{\alpha \beta \sigma \tau} \gamma_{\sigma \tau}\left(\boldsymbol{\zeta}^{m}(0)\right) \gamma_{\alpha \beta}\left(\boldsymbol{\zeta}^{m}(0)\right) \sqrt{a} \mathrm{~d} y \\
& \quad+\int_{0}^{t} \int_{\omega} p^{i, \varepsilon}(\tau) \dot{\zeta}_{i}^{m}(\tau) \sqrt{a} \mathrm{~d} y \mathrm{~d} \tau \\
& \leq C \rho \varepsilon^{3}\left\|\boldsymbol{\zeta}_{1}\right\|_{\boldsymbol{L}^{2}(\omega)}^{2}+\int_{0}^{t} \int_{\omega} p^{i, \varepsilon}(\tau) \dot{\zeta}_{i}^{m}(\tau) \sqrt{a} \mathrm{~d} y \mathrm{~d} \tau+C\left\|\boldsymbol{\zeta}_{0}\right\|_{\boldsymbol{V}_{K}(\omega)}^{2} \\
&+\frac{1}{2 \kappa} \int_{\omega} a^{\alpha \beta \sigma \tau} \gamma_{\sigma \tau}\left(\boldsymbol{\zeta}^{m}(0)\right) \gamma_{\alpha \beta}\left(\boldsymbol{\zeta}^{m}(0)\right) \sqrt{a} \mathrm{~d} y .
\end{aligned}
$$

Since each $p^{i, \varepsilon}$ is in $L^{\infty}\left(0, T ; L^{2}(\omega)\right)$, an application of Cauchy-Schwarz inequality gives

$$
\begin{aligned}
& \int_{0}^{t} \int_{\omega} p^{i, \varepsilon}(\tau) \dot{\zeta}_{i}^{m}(\tau) \sqrt{a} \mathrm{~d} y \mathrm{~d} \tau \leq\left(\int_{0}^{T}\left\|\boldsymbol{p}^{\varepsilon}(t)\right\|_{\boldsymbol{L}^{2}(\omega)}^{2} \mathrm{~d} t\right)^{1 / 2}\left(\int_{0}^{t}\left\|\dot{\boldsymbol{\zeta}}^{m}(\tau)\right\|_{\boldsymbol{L}^{2}(\omega)}^{2} \mathrm{~d} \tau\right)^{1 / 2} \\
& \leq \frac{1}{2}\left(\int_{0}^{T}\left\|\boldsymbol{p}^{\varepsilon}(t)\right\|_{\boldsymbol{L}^{2}(\omega)}^{2} \mathrm{~d} t+\int_{0}^{t}\left\|\dot{\boldsymbol{\zeta}}^{m}(\tau)\right\|_{\boldsymbol{L}^{2}(\omega)}^{2} \mathrm{~d} \tau\right) .
\end{aligned}
$$

Since $\gamma_{\alpha \beta}\left(\boldsymbol{\zeta}_{0}\right)=0$, and $\boldsymbol{\zeta}_{0}^{m} \rightarrow \boldsymbol{\zeta}_{0}$ in $\boldsymbol{V}_{K}(\omega)$, we have that

$$
\int_{\omega} a^{\alpha \beta \sigma \tau} \gamma_{\sigma \tau}\left(\boldsymbol{\zeta}_{0}^{m}\right) \gamma_{\alpha \beta}\left(\boldsymbol{\zeta}_{0}^{m}\right) \sqrt{a} \mathrm{~d} y \rightarrow 0, \text { as } m \rightarrow \infty .
$$

Prepared using sagej.cls 
As a result, there exists an integer $m(\kappa) \geq 1$ such that

$$
\int_{\omega} a^{\alpha \beta \sigma \tau} \gamma_{\sigma \tau}\left(\boldsymbol{\zeta}_{0}^{m}\right) \gamma_{\alpha \beta}\left(\boldsymbol{\zeta}_{0}^{m}\right) \sqrt{a} \mathrm{~d} y \leq \kappa,
$$

for all $m \geq m(\kappa)$. In conclusion, we obtain

$$
\frac{1}{2 \kappa} \int_{\omega} a^{\alpha \beta \sigma \tau} \gamma_{\sigma \tau}\left(\boldsymbol{\zeta}_{0}^{m}\right) \gamma_{\alpha \beta}\left(\boldsymbol{\zeta}_{0}^{m}\right) \sqrt{a} \mathrm{~d} y \leq \frac{1}{2} .
$$

By virtue of the uniform positive-definiteness of the elasticity tensor of the shell $\left(a^{\alpha \beta \sigma \tau}\right)$, and Korn inequality on a general surface (Theorem 3.1), for each integer $m \geq m(\kappa)$, there exists a real constant $\tilde{C}>0$ independent of $\boldsymbol{\zeta}^{m}$ (and so independent of $t, m$ and $\kappa$ ) for which the following estimate holds true

$$
\begin{aligned}
& \frac{1}{\tilde{C}}\left\{\left\|\dot{\boldsymbol{\zeta}}^{m}(t)\right\|_{\boldsymbol{L}^{2}(\omega)}^{2}+\left\|\boldsymbol{\zeta}^{m}(t)\right\|_{\boldsymbol{V}_{K}(\omega)}^{2}+\frac{1}{\kappa} \sum_{\alpha, \beta}\left\|\gamma_{\alpha \beta}\left(\boldsymbol{\zeta}^{m}(t)\right)\right\|_{0, \omega}^{2}\right\} \\
& \leq\left\|\boldsymbol{\zeta}_{1}\right\|_{\boldsymbol{L}^{2}(\omega)}^{2}+\left\|\boldsymbol{p}^{\varepsilon}\right\|_{L^{\infty}\left(0, T ; \boldsymbol{L}^{2}(\omega)\right)}^{2}+\left\|\boldsymbol{\zeta}_{0}\right\|_{\boldsymbol{V}_{K}(\omega)}^{2} \\
& \quad+\frac{1}{\kappa} \sum_{\alpha, \beta} \int_{0}^{t}\left\|\gamma_{\alpha \beta}\left(\boldsymbol{\zeta}^{m}(\tau)\right)\right\|_{0, \omega}^{2} \mathrm{~d} \tau \\
& \quad+\int_{0}^{t}\left\{\left\|\dot{\boldsymbol{\zeta}}^{m}(\tau)\right\|_{\boldsymbol{L}^{2}(\omega)}^{2}+\left\|\boldsymbol{\zeta}^{m}(\tau)\right\|_{\boldsymbol{V}_{K}(\omega)}^{2}\right\} \mathrm{d} \tau .
\end{aligned}
$$

An application of Gronwall's inequality (Theorem 3.2) with $a \equiv \tilde{C}>0$ and

$$
b \equiv \tilde{C}\left(\left\|\boldsymbol{\zeta}_{1}\right\|_{\boldsymbol{L}^{2}(\omega)}^{2}+\left\|\boldsymbol{p}^{\varepsilon}\right\|_{L^{\infty}\left(0, T ; \boldsymbol{L}^{2}(\omega)\right)}^{2}+\left\|\boldsymbol{\zeta}_{0}\right\|_{\boldsymbol{V}_{K}(\omega)}^{2}\right) \geq 0
$$

gives the following upper bound

$$
\begin{aligned}
& \int_{0}^{t}\left\{\left\|\dot{\boldsymbol{\zeta}}^{m}(\tau)\right\|_{\boldsymbol{L}^{2}(\omega)}^{2}+\left\|\boldsymbol{\zeta}^{m}(\tau)\right\|_{\boldsymbol{V}_{K}(\omega)}^{2}\right\} \mathrm{d} \tau+\frac{1}{\kappa} \sum_{\alpha, \beta} \int_{0}^{t}\left\|\gamma_{\alpha \beta}\left(\boldsymbol{\zeta}^{m}(\tau)\right)\right\|_{0, \omega}^{2} \mathrm{~d} \tau \\
& \leq \tilde{C} T e^{\tilde{C} T}\left\{\left\|\boldsymbol{\zeta}_{1}\right\|_{\boldsymbol{L}^{2}(\omega)}^{2}+\left\|\boldsymbol{p}^{\varepsilon}\right\|_{L^{\infty}\left(0, T ; \boldsymbol{L}^{2}(\omega)\right)}^{2}+\left\|\boldsymbol{\zeta}_{0}\right\|_{\boldsymbol{V}_{K}(\omega)}^{2}\right\}
\end{aligned}
$$

for all $t \in[0, T]$.

Therefore, we obtain that

$\left(\boldsymbol{\zeta}^{m}\right)_{m=1}^{\infty}$ is uniformly bounded with respect to $m$ in $L^{\infty}\left(0, T ; \boldsymbol{V}_{K}(\omega)\right)$,

$\left(\dot{\boldsymbol{\zeta}}^{m}\right)_{m=1}^{\infty}$ is uniformly bounded with respect to $m$ in $L^{\infty}\left(0, T ; \boldsymbol{L}^{2}(\omega)\right)$.

Moreover, by (4), there exists a constant $L>0$, independent of $m, \kappa$ and $t$, such that

$$
0 \leq\left\|\tilde{\gamma}_{\alpha \beta}\left(\boldsymbol{\zeta}^{m}\right)\right\|_{L^{2}\left(0, T ; L^{2}(\omega)\right)}^{2} \leq L \kappa
$$

Prepared using sagej.cls 
Since the following direct sum decomposition holds

$$
\boldsymbol{V}_{K}(\omega)=\boldsymbol{E}^{m} \oplus\left(\boldsymbol{E}^{m}\right)^{\perp}
$$

and since $\ddot{\boldsymbol{\zeta}}^{m}(t) \in \boldsymbol{E}^{m}$, the variational equations of Problem $\mathcal{P}_{F, \kappa}^{\varepsilon, m}(\omega)$ give the existence of a constant $C_{\kappa}>0$, independent of $m$ and $t$, such that

$$
\left|\int_{\omega} \ddot{\zeta}_{i}^{m}(t) \eta_{i} \sqrt{a} \mathrm{~d} y\right| \leq C_{\kappa}\left(\left\|\boldsymbol{p}^{\varepsilon}\right\|_{L^{\infty}\left(0, T ; \boldsymbol{L}^{2}(\omega)\right)}+\left\|\boldsymbol{\zeta}^{m}\right\|_{L^{\infty}\left(0, T ; \boldsymbol{V}_{K}(\omega)\right)}\right),
$$

for any $\boldsymbol{\eta} \in \boldsymbol{V}_{K}(\omega)$, with $\|\boldsymbol{\eta}\|_{\boldsymbol{V}_{K}(\omega)} \leq 1$, and a.a. $t \in(0, T)$. As a consequence of (5) we have

$$
\left\|\ddot{\boldsymbol{\zeta}}^{m}\right\|_{L^{\infty}\left(0, T ; \boldsymbol{V}_{K}^{*}(\omega)\right)} \leq C_{\kappa} .
$$

(iii) Passage to the limit and retrieval of Problem $\mathcal{P}_{F, \kappa}^{\varepsilon}(\omega)$. By (5) and (7), we can infer that there exist subsequences, still denoted $\left(\boldsymbol{\zeta}^{m}\right)_{m=1}^{\infty},\left(\dot{\zeta}^{m}\right)_{m=1}^{\infty}$ and $\left(\ddot{\zeta}^{m}\right)_{m=1}^{\infty}$ such that the following convergences take place:

$$
\begin{array}{ll}
\boldsymbol{\zeta}^{m} \stackrel{*}{\rightarrow} \boldsymbol{\zeta}_{\kappa}^{\varepsilon}, & \text { in } L^{\infty}\left(0, T ; \boldsymbol{V}_{K}(\omega)\right) \text { as } m \rightarrow \infty, \\
\dot{\boldsymbol{\zeta}}^{m} \stackrel{*}{*} \dot{\boldsymbol{\zeta}}_{\kappa}^{\varepsilon}, & \text { in } L^{\infty}\left(0, T ; \boldsymbol{L}^{2}(\omega)\right) \text { as } m \rightarrow \infty, \\
\ddot{\boldsymbol{\zeta}}^{m} \stackrel{*}{\rightarrow} \ddot{\boldsymbol{\zeta}}_{\kappa}^{\varepsilon}, & \text { in } L^{\infty}\left(0, T ; \boldsymbol{V}_{K}^{*}(\omega)\right) \text { as } m \rightarrow \infty .
\end{array}
$$

Observe that, by Corollary 8.18 of [23], the following convergence also holds

$$
\boldsymbol{\zeta}^{m} \rightarrow \boldsymbol{\zeta}_{\kappa}^{\varepsilon}, \quad \text { in } L^{2}\left(0, T ; \boldsymbol{V}_{K}(\omega)\right) \text { as } m \rightarrow \infty,
$$

the space $\boldsymbol{V}_{K}(\omega)$ being reflexive.

By Sobolev embedding theorem (Theorem 10.1.25 of [35]), we obtain

$$
\begin{array}{ll}
\boldsymbol{\zeta}^{m} \rightarrow \boldsymbol{\zeta}_{\kappa}^{\varepsilon}, & \text { in } \mathcal{C}^{0}\left([0, T] ; \boldsymbol{L}^{2}(\omega)\right), \\
\dot{\boldsymbol{\zeta}}^{m} \rightarrow \dot{\boldsymbol{\zeta}}_{\kappa}^{\varepsilon}, & \text { in } \mathcal{C}^{0}\left([0, T] ; \boldsymbol{V}_{K}^{*}(\omega)\right) .
\end{array}
$$

We now verify that $\boldsymbol{\zeta}_{\kappa}^{\varepsilon}$ is a weak solution of the variational equations of Problem $\mathcal{P}_{F, \kappa}^{\varepsilon}(\omega)$. Let $\psi \in \mathcal{D}(0, T)$ and let $\mu \geq 1$ be any integer. For each $m \geq \mu$, the variational equations of Problem $\mathcal{P}_{F, \kappa}^{\varepsilon, m}(\omega)$ give

$$
\begin{aligned}
& 2 \rho \varepsilon^{3} \int_{0}^{T} \int_{\omega} \ddot{\zeta}_{i}^{m}(t) \eta_{i} \sqrt{a} \mathrm{~d} y \psi(t) \mathrm{d} t \\
& \quad+\frac{\varepsilon^{3}}{3} \int_{0}^{T} \int_{\omega} a^{\alpha \beta \sigma \tau} \rho_{\sigma \tau}\left(\boldsymbol{\zeta}^{m}(t)\right) \rho_{\alpha \beta}(\boldsymbol{\eta}) \sqrt{a} \mathrm{~d} y \psi(t) \mathrm{d} t \\
& \quad+\frac{1}{\kappa} \int_{0}^{T} \int_{\omega} a^{\alpha \beta \sigma \tau} \gamma_{\sigma \tau}\left(\boldsymbol{\zeta}^{m}(t)\right) \gamma_{\alpha \beta}(\boldsymbol{\eta}) \sqrt{a} \mathrm{~d} y \psi(t) \mathrm{d} t \\
& =\int_{0}^{T} \int_{\omega} p^{i, \varepsilon}(t) \eta_{i} \sqrt{a} \mathrm{~d} y \psi(t) \mathrm{d} t,
\end{aligned}
$$

for all $\boldsymbol{\eta} \in \boldsymbol{E}^{\mu}$.

Prepared using sagej.cls 
Consider the real-valued mapping

$$
\begin{aligned}
\boldsymbol{\xi} \in L^{2}\left(0, T ; \boldsymbol{V}_{K}(\omega)\right) \rightarrow \int_{0}^{T} & \int_{\omega} a^{\alpha \beta \sigma \tau} \tilde{\rho}_{\sigma \tau}(\boldsymbol{\xi})(t) \rho_{\alpha \beta}(\boldsymbol{\eta}) \sqrt{a} \mathrm{~d} y \psi(t) \mathrm{d} t \\
& +\frac{1}{\kappa} \int_{0}^{T} \int_{\omega} a^{\alpha \beta \sigma \tau} \tilde{\gamma}_{\sigma \tau}(\boldsymbol{\xi})(t) \gamma_{\alpha \beta}(\boldsymbol{\eta}) \sqrt{a} \mathrm{~d} y \psi(t) \mathrm{d} t
\end{aligned}
$$

and observe that it is linear and continuous, as a consequence of the linearity and continuity of $\tilde{\rho}_{\alpha \beta}$ and $\tilde{\gamma}_{\alpha \beta}$.

The convergence process (8) thus gives

$$
\begin{aligned}
\int_{0}^{T} & \int_{\omega} a^{\alpha \beta \sigma \tau} \rho_{\sigma \tau}\left(\boldsymbol{\zeta}^{m}(t)\right) \rho_{\alpha \beta}(\boldsymbol{\eta}) \sqrt{a} \mathrm{~d} y \psi(t) \mathrm{d} t \\
& +\frac{1}{\kappa} \int_{0}^{T} \int_{\omega} a^{\alpha \beta \sigma \tau} \gamma_{\sigma \tau}\left(\boldsymbol{\zeta}^{m}(t)\right) \gamma_{\alpha \beta}(\boldsymbol{\eta}) \sqrt{a} \mathrm{~d} y \psi(t) \mathrm{d} t \\
= & \int_{0}^{T} \int_{\omega} a^{\alpha \beta \sigma \tau} \tilde{\rho}_{\sigma \tau}\left(\boldsymbol{\zeta}^{m}\right)(t) \rho_{\alpha \beta}(\boldsymbol{\eta}) \sqrt{a} \mathrm{~d} y \psi(t) \mathrm{d} t \\
& +\frac{1}{\kappa} \int_{0}^{T} \int_{\omega} a^{\alpha \beta \sigma \tau} \tilde{\gamma}_{\sigma \tau}\left(\boldsymbol{\zeta}^{m}\right)(t) \gamma_{\alpha \beta}(\boldsymbol{\eta}) \sqrt{a} \mathrm{~d} y \psi(t) \mathrm{d} t \\
\rightarrow & \int_{0}^{T} \int_{\omega} a^{\alpha \beta \sigma \tau} \rho_{\sigma \tau}\left(\boldsymbol{\zeta}_{\kappa}^{\varepsilon}(t)\right) \rho_{\alpha \beta}(\boldsymbol{\eta}) \sqrt{a} \mathrm{~d} y \psi(t) \mathrm{d} t \\
& +\frac{1}{\kappa} \int_{0}^{T} \int_{\omega} a^{\alpha \beta \sigma \tau} \gamma_{\sigma \tau}\left(\boldsymbol{\zeta}_{\kappa}^{\varepsilon}(t)\right) \gamma_{\alpha \beta}(\boldsymbol{\eta}) \sqrt{a} \mathrm{~d} y \psi(t) \mathrm{d} t .
\end{aligned}
$$

Observe, also, that the following density holds

$$
\bigcup_{\mu \geq 1} \boldsymbol{E}^{\mu \cdot \|_{V_{K}(\omega)}}=\boldsymbol{V}_{K}(\omega),
$$

As a result, keeping in mind the convergence processes (8) and (11), and letting $m \rightarrow \infty$ in (10) gives that $\boldsymbol{\zeta}_{\kappa}^{\varepsilon}$ is a solution of the following variational equations

$$
\begin{aligned}
& 2 \rho \varepsilon^{3} \boldsymbol{V}_{K}^{*}\left\langle\ddot{\boldsymbol{\zeta}}_{\kappa}^{\varepsilon}(t), \boldsymbol{\eta}\right\rangle_{\boldsymbol{V}_{K}(\omega)} \\
& \quad+\frac{\varepsilon^{3}}{3} \int_{\omega} a^{\alpha \beta \sigma \tau} \rho_{\sigma \tau}\left(\boldsymbol{\zeta}_{\kappa}^{\varepsilon}(t)\right) \rho_{\alpha \beta}(\boldsymbol{\eta}) \sqrt{a} \mathrm{~d} y \\
& \quad+\frac{1}{\kappa} \int_{\omega} a^{\alpha \beta \sigma \tau} \gamma_{\sigma \tau}\left(\boldsymbol{\zeta}_{\kappa}^{\varepsilon}(t)\right) \gamma_{\alpha \beta}(\boldsymbol{\eta}) \sqrt{a} \mathrm{~d} y \\
& =\int_{\omega} p^{i, \varepsilon}(t) \eta_{i} \sqrt{a} \mathrm{~d} y,
\end{aligned}
$$

for all $\boldsymbol{\eta} \in \boldsymbol{V}_{K}(\omega)$, in the sense of distributions in $(0, T)$. Since $\boldsymbol{\zeta}_{\kappa}^{\varepsilon}(t) \in$ $\boldsymbol{V}_{K}(\omega)$ for a.a. $t \in(0, T)$ and since $\boldsymbol{\eta} \in \boldsymbol{V}_{K}(\omega)$ is independent of the time variable $t$, two consecutive applications of the integration by parts formula (cf.

Prepared using sagej.cls 
Corollary 10.1.26 of [35]) give

$$
\frac{\mathrm{d}^{2}}{\mathrm{~d} t^{2}} \int_{\omega} \zeta_{i, \kappa}^{\varepsilon}(t) \eta_{i} \sqrt{a} \mathrm{~d} y=\boldsymbol{V}_{K}^{*}\left\langle\ddot{\zeta}_{\kappa}^{\varepsilon}(t), \boldsymbol{\eta}\right\rangle_{\boldsymbol{V}_{K}(\omega)}, \text { for a.a. } t \in(0, T),
$$

showing that $\boldsymbol{\zeta}_{\kappa}^{\varepsilon}$ satisfies the variational equations of Problem $\mathcal{P}_{F, \kappa}^{\varepsilon}(\omega)$ in the sense of distributions in $(0, T)$.

The last thing that we have to check is the validity of the initial conditions for $\boldsymbol{\zeta}_{\kappa}^{\varepsilon}$.

Let us introduce the operator $\boldsymbol{L}_{0}: \mathcal{C}^{0}\left([0, T] ; \boldsymbol{L}^{2}(\omega)\right) \rightarrow \boldsymbol{L}^{2}(\omega)$ defined in a way such that $\boldsymbol{L}_{0}(\boldsymbol{\eta}):=\boldsymbol{\eta}(0)$. Such an operator $\boldsymbol{L}_{0}$ turns out to be linear and continuous and, therefore, by the first convergence of (9), we get that

$$
\boldsymbol{\zeta}_{0}^{m}=\boldsymbol{\zeta}^{m}(0) \rightarrow \boldsymbol{\zeta}_{\kappa}^{\varepsilon}(0), \quad \text { in } \boldsymbol{L}^{2}(\omega) .
$$

Since $\boldsymbol{\zeta}_{0}^{m} \rightarrow \boldsymbol{\zeta}_{0}$ in $\boldsymbol{V}_{K}(\omega)$, we deduce that $\boldsymbol{\zeta}_{\kappa}^{\varepsilon}(0)=\boldsymbol{\zeta}_{0}$.

Let us introduce the operator $\boldsymbol{L}_{1}: \mathcal{C}^{0}\left([0, T] ; \boldsymbol{V}_{K}^{*}(\omega)\right) \rightarrow \boldsymbol{V}_{K}^{*}(\omega)$ defined in a way such that $\boldsymbol{L}_{1}(\boldsymbol{\eta}):=\boldsymbol{\eta}(0)$. Such an operator $\boldsymbol{L}_{1}$ turns out to be linear and continuous and, therefore, by the second convergence of (9), we get that

$$
\boldsymbol{\zeta}_{1}^{m}=\dot{\boldsymbol{\zeta}}^{m}(0) \rightarrow \dot{\boldsymbol{\zeta}}_{\kappa}^{\varepsilon}(0), \quad \text { in } \boldsymbol{V}_{K}^{*}(\omega) .
$$

Since $\boldsymbol{\zeta}_{1}^{m} \rightarrow \boldsymbol{\zeta}_{1}$ in $\boldsymbol{L}^{2}(\omega)$, we deduce that $\dot{\boldsymbol{\zeta}}_{\kappa}^{\varepsilon}(0)=\boldsymbol{\zeta}_{1}$. The existence of a weak solution of Problem $\mathcal{P}_{F, \kappa}^{\varepsilon}(\omega)$ has thus been shown.

(iv) The weak solution $\boldsymbol{\zeta}_{\kappa}^{\varepsilon}$ is actually strong and uniquely determined. Recall that the bilinear form $a_{\kappa}(\cdot, \cdot)$ is symmetric, continuous, and $\boldsymbol{V}_{K}(\omega)$-elliptic and that the space $\boldsymbol{V}_{K}(\omega)$ is continuously and densely embedded in $\boldsymbol{L}^{2}(\omega)$. We are thus in a position to apply the same procedure presented in Theorem 8.2-2 of [31].

Let us also observe that the convergence $\boldsymbol{\zeta}^{m} \rightarrow \boldsymbol{\zeta}_{\kappa}^{\varepsilon}$ in $\mathcal{C}^{0}\left([0, T] ; \boldsymbol{V}_{K}(\omega)\right)$ gives

$$
\tilde{\gamma}_{\alpha \beta}\left(\boldsymbol{\zeta}^{m}\right) \rightarrow \tilde{\gamma}_{\alpha \beta}\left(\boldsymbol{\zeta}_{\kappa}^{\varepsilon}\right), \quad \text { in } L^{2}\left(0, T ; L^{2}(\omega)\right),
$$

and so, by (6), the following energy estimate

$$
\left\|\tilde{\gamma}_{\alpha \beta}\left(\boldsymbol{\zeta}_{\kappa}^{\varepsilon}\right)\right\|_{L^{2}\left(0, T ; L^{2}(\omega)\right)} \leq \sqrt{L \kappa} .
$$

This completes the proof.

Noticeably, unlike Section 4, the existence and uniqueness of weak solutions for Problem $\mathcal{P}_{F}^{\varepsilon}(\omega)$ cannot be directly carried out via Galerkin method, since the space $\boldsymbol{V}_{F}(\omega)$ is not, in general, dense in $\boldsymbol{L}^{2}(\omega)$. This fact prevents us from applying Theorem $6.2-1$ of [31] directly.

\section{The main result: Existence and uniqueness of solutions of Problem $\mathcal{P}_{F}^{\varepsilon}(\omega)$}

We are now ready to prove the main theoretical result of this paper: the existence and uniqueness of weak solutions of Problem $\mathcal{P}_{F}^{\varepsilon}(\omega)$.

Prepared using sagej.cls 
Theorem 6.1. Problem $\mathcal{P}_{F}^{\varepsilon}(\omega)$ admits a unique weak solution $\boldsymbol{\zeta}^{\varepsilon}$.

Proof. (i) Problem $\mathcal{P}_{F}^{\varepsilon}(\omega)$ admits a weak solution. By the energy estimates (4) in Theorem 5.1 and the fact that $\boldsymbol{\zeta}_{0} \in \boldsymbol{V}_{F}(\omega)$, it can be easily observed that there exists a positive constant $c=c\left(\boldsymbol{\zeta}_{0}, \boldsymbol{\zeta}_{1}, \boldsymbol{p}^{\varepsilon}\right)$ such that

$$
\left\{\left\|\dot{\boldsymbol{\zeta}}_{\kappa}^{\varepsilon}\right\|_{L^{\infty}\left(0, T ; \boldsymbol{L}^{2}(\omega)\right)}^{2}+\left\|\boldsymbol{\zeta}_{\kappa}^{\varepsilon}\right\|_{L^{\infty}\left(0, T ; \boldsymbol{V}_{K}(\omega)\right)}^{2}\right\} \leq c .
$$

Let us consider, for a.a. $0<t<T$, the following partial differential equation associated with Problem $\mathcal{P}_{F, \kappa}^{\varepsilon}(\omega)$

$$
2 \rho \varepsilon^{3} \ddot{\boldsymbol{\zeta}}_{\kappa}^{\varepsilon}(t)+A \boldsymbol{\zeta}_{\kappa}^{\varepsilon}(t)+\frac{1}{\kappa} P \boldsymbol{\zeta}_{\kappa}^{\varepsilon}(t)=\boldsymbol{p}^{\varepsilon}(t), \quad \text { in } \boldsymbol{V}_{K}^{*}(\omega),
$$

where the linear operator $A: \boldsymbol{V}_{K}(\omega) \rightarrow \boldsymbol{V}_{K}^{*}(\omega)$ defined by

$\boldsymbol{V}_{K}^{*}(\omega)\langle A \boldsymbol{\xi}, \boldsymbol{\eta}\rangle_{\boldsymbol{V}_{K}(\omega)}:=\frac{\varepsilon^{3}}{3} \int_{\omega} a^{\alpha \beta \sigma \tau} \rho_{\sigma \tau}(\boldsymbol{\xi}) \rho_{\alpha \beta}(\boldsymbol{\eta}) \sqrt{a} \mathrm{~d} y, \quad$ for all $\boldsymbol{\xi}, \boldsymbol{\eta} \in \boldsymbol{V}_{K}(\omega)$,

is linear and continuous. Similarly, the operator $P$ defined by

$$
\boldsymbol{V}_{K}^{*}(\omega)\langle P \boldsymbol{\xi}, \boldsymbol{\eta}\rangle_{\boldsymbol{V}_{K}(\omega)}:=\int_{\omega} a^{\alpha \beta \sigma \tau} \gamma_{\sigma \tau}(\boldsymbol{\xi}) \gamma_{\alpha \beta}(\boldsymbol{\eta}) \sqrt{a} \mathrm{~d} y, \quad \text { for all } \boldsymbol{\xi}, \boldsymbol{\eta} \in \boldsymbol{V}_{K}(\omega)
$$

is linear and continuous. Besides, for all $\boldsymbol{\eta} \in \boldsymbol{V}_{F}(\omega)$, we have

$$
\boldsymbol{V}_{K}^{*}(\omega)\left\langle P \boldsymbol{\zeta}_{\kappa}^{\varepsilon}, \boldsymbol{\eta}\right\rangle_{\boldsymbol{V}_{K}(\omega)}=0 .
$$

As a result, the family $\left(\ddot{\boldsymbol{\zeta}}_{\kappa}^{\varepsilon}\right)_{\kappa>0}$ is uniformly bounded in $L^{\infty}\left(0, T ; \boldsymbol{V}_{F}^{*}(\omega)\right)$. Hence, up to passing to a subsequence, we get that the following convergence process takes place

$$
\begin{array}{ll}
\boldsymbol{\zeta}_{\kappa}^{\varepsilon} \stackrel{*}{\rightarrow}^{\varepsilon}, & \text { in } L^{\infty}\left(0, T ; \boldsymbol{V}_{K}(\omega)\right) \text { as } \kappa \rightarrow 0, \\
\dot{\boldsymbol{\zeta}}_{\kappa}^{\varepsilon} \stackrel{*}{\rightarrow} \dot{\boldsymbol{\zeta}}^{\varepsilon}, & \text { in } L^{\infty}\left(0, T ; \boldsymbol{L}^{2}(\omega)\right) \text { as } \kappa \rightarrow 0, \\
\ddot{\boldsymbol{\zeta}}_{\kappa}^{\varepsilon} \stackrel{*}{\boldsymbol{\zeta}}^{\varepsilon}, & \text { in } L^{\infty}\left(0, T ; \boldsymbol{V}_{F}^{*}(\omega)\right) \text { as } \kappa \rightarrow 0
\end{array}
$$

By Sobolev embedding theorem (Theorem 10.1.25 of [35]), we have that

$$
\begin{array}{ll}
\boldsymbol{\zeta}_{\kappa}^{\varepsilon} \rightarrow \boldsymbol{\zeta}^{\varepsilon}, & \text { in } \mathcal{C}^{0}\left([0, T] ; \boldsymbol{L}^{2}(\omega)\right), \\
\dot{\boldsymbol{\zeta}}_{\kappa}^{\varepsilon} \rightarrow \dot{\boldsymbol{\zeta}}^{\varepsilon}, & \text { in } \mathcal{C}^{0}\left([0, T] ; \boldsymbol{V}_{F}^{*}(\omega)\right) .
\end{array}
$$

Let us recall that (see (13)), there exists a constant $L>0$, independent of $\kappa$ and $t$, such that

$$
\left\|\tilde{\gamma}_{\alpha \beta}\left(\boldsymbol{\zeta}_{\kappa}^{\varepsilon}\right)\right\|_{L^{2}\left(0, T ; L^{2}(\omega)\right)} \leq \sqrt{L \kappa} .
$$

and observe that the convergence process (14) gives

$$
\tilde{\gamma}_{\alpha \beta}\left(\boldsymbol{\zeta}_{\kappa}^{\varepsilon}\right) \rightarrow \tilde{\gamma}_{\alpha \beta}\left(\boldsymbol{\zeta}^{\varepsilon}\right), \quad \text { in } L^{2}\left(0, T ; L^{2}(\omega)\right)
$$

Prepared using sagej.cls 
and so, by the energy estimate (13),

$$
\left\|\tilde{\gamma}_{\alpha \beta}\left(\boldsymbol{\zeta}^{\varepsilon}\right)\right\|_{L^{2}\left(0, T ; L^{2}(\omega)\right)} \leq \liminf _{\kappa \rightarrow 0}\left\|\tilde{\gamma}_{\alpha \beta}\left(\boldsymbol{\zeta}_{\kappa}^{\varepsilon}\right)\right\|_{L^{2}\left(0, T ; L^{2}(\omega)\right)}=0 .
$$

In conclusion, by the definition of $\tilde{\gamma}_{\alpha \beta}$, we get that $\gamma_{\alpha \beta}\left(\boldsymbol{\zeta}^{\varepsilon}(t)\right)=0$ in $\omega$ for a.a. $t \in(0, T)$ and we can thus gain more insight into the regularity of $\boldsymbol{\zeta}^{\varepsilon}$, viz.,

$$
\boldsymbol{\zeta}^{\varepsilon}(t) \in \boldsymbol{V}_{F}(\omega), \quad \text { for a.a. } 0<t<T .
$$

Let us show that $\boldsymbol{\zeta}^{\varepsilon}$ is a weak solution of Problem $\mathcal{P}_{F}^{\varepsilon}(\omega)$. We first show that $\boldsymbol{\zeta}^{\varepsilon}$ solves the variational equations of Problem $\mathcal{P}_{F}^{\varepsilon}(\omega)$ in the sense of distributions in $(0, T)$. For each $\psi \in \mathcal{D}(0, T)$ and each $\boldsymbol{\eta} \in \boldsymbol{V}_{F}(\omega)$, the variational equations of Problem $\mathcal{P}_{F, \kappa}^{\varepsilon}(\omega)$ give

$$
\begin{aligned}
& 2 \rho \varepsilon^{3} \int_{0}^{T} \boldsymbol{V}_{F}^{*}(\omega)\left\langle\ddot{\boldsymbol{\zeta}}_{\kappa}^{\varepsilon}(t), \boldsymbol{\eta}\right\rangle_{\boldsymbol{V}_{F}(\omega)} \psi(t) \mathrm{d} t \\
& \quad+\frac{\varepsilon^{3}}{3} \int_{0}^{T} \int_{\omega} a^{\alpha \beta \sigma \tau} \rho_{\sigma \tau}\left(\boldsymbol{\zeta}_{\kappa}^{\varepsilon}(t)\right) \rho_{\alpha \beta}(\boldsymbol{\eta}) \sqrt{a} \mathrm{~d} y \psi(t) \mathrm{d} t \\
& \quad+\frac{1}{\kappa} \int_{0}^{T} \int_{\omega} a^{\alpha \beta \sigma \tau} \gamma_{\sigma \tau}\left(\boldsymbol{\zeta}_{\kappa}^{\varepsilon}(t)\right) \gamma_{\alpha \beta}(\boldsymbol{\eta}) \sqrt{a} \mathrm{~d} y \psi(t) \mathrm{d} t \\
& =\int_{0}^{T} \int_{\omega} p^{i, \varepsilon}(t) \eta_{i} \sqrt{a} \mathrm{~d} y \psi(t) \mathrm{d} t .
\end{aligned}
$$

Since $\boldsymbol{\eta} \in \boldsymbol{V}_{F}(\omega)$, we have

$$
\frac{1}{\kappa} \int_{\omega} a^{\alpha \beta \sigma \tau} \gamma_{\sigma \tau}\left(\boldsymbol{\zeta}_{\kappa}^{\varepsilon}(t)\right) \gamma_{\alpha \beta}(\boldsymbol{\eta}) \sqrt{a} \mathrm{~d} y=0,
$$

for all $\kappa>0$ and a.a. $t \in(0, T)$.

Consider the real-valued mapping

$$
\boldsymbol{\xi} \in L^{2}\left(0, T ; \boldsymbol{V}_{K}(\omega)\right) \rightarrow \int_{0}^{T} \int_{\omega} a^{\alpha \beta \sigma \tau} \tilde{\rho}_{\sigma \tau}(\boldsymbol{\xi})(t) \rho_{\alpha \beta}(\boldsymbol{\eta}) \sqrt{a} \mathrm{~d} y \psi(t) \mathrm{d} t,
$$

and observe that it is linear and continuous, as a consequence of the linearity and continuity of $\tilde{\rho}_{\alpha \beta}$.

The convergence process (14) thus gives

$$
\begin{aligned}
& \int_{0}^{T} \int_{\omega} a^{\alpha \beta \sigma \tau} \rho_{\sigma \tau}\left(\boldsymbol{\zeta}_{\kappa}^{\varepsilon}(t)\right) \rho_{\alpha \beta}(\boldsymbol{\eta}) \sqrt{a} \mathrm{~d} y \psi(t) \mathrm{d} t \\
= & \int_{0}^{T} \int_{\omega} a^{\alpha \beta \sigma \tau} \tilde{\rho}_{\sigma \tau}\left(\boldsymbol{\zeta}_{\kappa}^{\varepsilon}\right)(t) \rho_{\alpha \beta}(\boldsymbol{\eta}) \sqrt{a} \mathrm{~d} y \psi(t) \mathrm{d} t \\
\rightarrow & \int_{0}^{T} \int_{\omega} a^{\alpha \beta \sigma \tau} \rho_{\sigma \tau}\left(\boldsymbol{\zeta}^{\varepsilon}(t)\right) \rho_{\alpha \beta}(\boldsymbol{\eta}) \sqrt{a} \mathrm{~d} y \psi(t) \mathrm{d} t .
\end{aligned}
$$

Since $\boldsymbol{\zeta}^{\varepsilon} \in L^{\infty}\left(0, T ; \boldsymbol{V}_{F}(\omega)\right)$ and since $\boldsymbol{\eta} \in \boldsymbol{V}_{F}(\omega)$ is independent of the time variable $t$, two consecutive applications of the integration by parts formula (see 
Corollary 10.1.26 of [35]) give

$$
\frac{\mathrm{d}^{2}}{\mathrm{~d} t^{2}} \int_{\omega} \zeta_{i}^{\varepsilon}(t) \eta_{i} \sqrt{a} \mathrm{~d} y=\boldsymbol{V}_{F}^{*}(\omega)\left\langle\ddot{\boldsymbol{\zeta}}^{\varepsilon}(t), \boldsymbol{\eta}\right\rangle_{\boldsymbol{V}_{F}(\omega)} \text {, for a.a. } t \in(0, T),
$$

and we thus conclude that $\boldsymbol{\zeta}^{\varepsilon}$ solves the variational equations of Problem $\mathcal{P}_{F}^{\varepsilon}(\omega)$ in the sense of distributions in $(0, T)$.

The last thing to check is the validity of the initial conditions for $\boldsymbol{\zeta}^{\varepsilon}$. Let us introduce the operator $\boldsymbol{L}_{0}: \mathcal{C}^{0}\left([0, T] ; \boldsymbol{L}^{2}(\omega)\right) \rightarrow \boldsymbol{L}^{2}(\omega)$ defined in a way such that $\boldsymbol{L}_{0}(\boldsymbol{\eta}):=\boldsymbol{\eta}(0)$. Such an operator turns out to be linear and continuous and, therefore, by the convergence process (15), we get that

$$
\boldsymbol{\zeta}_{\kappa}^{\varepsilon}(0) \rightarrow \boldsymbol{\zeta}^{\varepsilon}(0)=\boldsymbol{\zeta}_{0}, \quad \text { in } \boldsymbol{L}^{2}(\omega)
$$

Let us introduce the operator $\boldsymbol{L}_{1}: \mathcal{C}^{0}\left([0, T] ; \boldsymbol{V}_{F}^{*}(\omega)\right) \rightarrow \boldsymbol{V}_{F}^{*}(\omega)$ defined in a way such that $\boldsymbol{L}_{1}(\boldsymbol{\eta}):=\boldsymbol{\eta}(0)$. Such an operator turns out to be linear and continuous and, therefore, by the convergence process (15), we get that

$$
\dot{\boldsymbol{\zeta}}_{\kappa}^{\varepsilon}(0) \rightarrow \dot{\boldsymbol{\zeta}}^{\varepsilon}(0)=\boldsymbol{\zeta}_{1}, \quad \text { in } \boldsymbol{V}_{F}^{*}(\omega) .
$$

The existence of a weak solution of Problem $\mathcal{P}_{F}^{\varepsilon}(\omega)$ has thus been shown.

(ii) The vector valued function $\boldsymbol{\zeta}^{\varepsilon}$ is the unique weak solution of Problem $\mathcal{P}_{F}^{\varepsilon}(\omega)$. Following the same strategy as for the wave equation (cf. [36]), let us show that the only weak solution of the initial value problem

$$
\begin{aligned}
& 2 \rho \varepsilon^{3} \ddot{\boldsymbol{\zeta}}^{\varepsilon}(t)+A \boldsymbol{\zeta}^{\varepsilon}(t)=\mathbf{0}, \quad \text { in } \boldsymbol{V}_{F}^{*}(\omega), \text { for a.a. } 0<t<T, \\
& \boldsymbol{\zeta}^{\varepsilon}(0)=\mathbf{0}, \\
& \dot{\boldsymbol{\zeta}}^{\varepsilon}(0)=\mathbf{0},
\end{aligned}
$$

is $\boldsymbol{\zeta}^{\varepsilon} \equiv \mathbf{0}$.

To this end, for any fixed $0 \leq s \leq T$, let us define the function

$$
\boldsymbol{\xi}(t):= \begin{cases}\int_{t}^{s} \boldsymbol{\zeta}^{\varepsilon}(\tau) \mathrm{d} \tau & , 0 \leq t \leq s, \\ \mathbf{0} & , s<t \leq T,\end{cases}
$$

and observe that (cf., e.g., Theorem 8.13 of $[23]) \boldsymbol{\xi} \in \mathcal{C}^{0}\left([0, T] ; \boldsymbol{V}_{F}(\omega)\right)$. Define the bilinear form $B_{F}: \boldsymbol{V}_{K}(\omega) \times \boldsymbol{V}_{K}(\omega) \rightarrow \mathbb{R}$ associated with the "flexural" part by

$$
B_{F}(\boldsymbol{\xi}, \boldsymbol{\eta}):=\frac{\varepsilon^{3}}{3} \int_{\omega} a^{\alpha \beta \sigma \tau} \rho_{\sigma \tau}(\boldsymbol{\xi}) \rho_{\alpha \beta}(\boldsymbol{\eta}) \sqrt{a} \mathrm{~d} y .
$$

Since $\dot{\boldsymbol{\zeta}}^{\varepsilon}(0)=\mathbf{0}=\boldsymbol{\xi}(s)$, an application of the integration by parts formula (Corollary 10.1.26 of [35]) yields

$$
\int_{0}^{s}\left\{-2 \rho \varepsilon^{3} \int_{\omega} \dot{\zeta}_{i}^{\varepsilon}(t) \dot{\xi}_{i}(t) \sqrt{a} \mathrm{~d} y+B_{F}\left(\boldsymbol{\zeta}^{\varepsilon}(t), \boldsymbol{\xi}(t)\right)\right\} \mathrm{d} t=0 .
$$

Prepared using sagej.cls 
Since $\dot{\boldsymbol{\xi}}(t)=-\boldsymbol{\zeta}^{\varepsilon}(t)$, for all $0 \leq t \leq s$, the latter formula becomes

$$
\int_{0}^{s}\left\{2 \rho \varepsilon^{3} \int_{\omega} \dot{\zeta}_{i}^{\varepsilon}(t) \zeta_{i}^{\varepsilon}(t) \sqrt{a} \mathrm{~d} y-B_{F}(\dot{\boldsymbol{\xi}}(t), \boldsymbol{\xi}(t))\right\} \mathrm{d} t=0 .
$$

Another application of integration by parts formula (Corollary 10.1.26 of [35]) transforms the latter into

$$
\int_{0}^{s} \frac{\mathrm{d}}{\mathrm{d} t}\left(\rho \varepsilon^{3}\left\|\boldsymbol{\zeta}^{\varepsilon}(t)\right\|_{\boldsymbol{L}^{2}(\omega)}^{2}-\frac{1}{2} B_{F}(\boldsymbol{\xi}(t), \boldsymbol{\xi}(t))\right) \mathrm{d} t=0,
$$

and the initial conditions in (16) give

$$
\rho \varepsilon^{3}\left\|\boldsymbol{\zeta}^{\varepsilon}(s)\right\|_{\boldsymbol{L}^{2}(\omega)}^{2}+\frac{1}{2} B_{F}(\boldsymbol{\xi}(0), \boldsymbol{\xi}(0))=0 .
$$

In conclusion, we have $B_{F}(\boldsymbol{\xi}(0), \boldsymbol{\xi}(0))=0$ and $\left\|\boldsymbol{\zeta}^{\varepsilon}(s)\right\|_{\boldsymbol{L}^{2}(\omega)}=0$, for all $0 \leq$ $s \leq T$. By the arbitrariness of $s$, we conclude that the weak solution $\boldsymbol{\zeta}^{\varepsilon}$ is uniquely defined almost everywhere in $(0, T)$. This completes the proof.

\section{Final considerations: The dynamics of elliptic membrane shells}

Consider a linearly elastic shell, subjected to the various assumptions set forth in Section 3. Following the terminology proposed in Section 4.1 of [21], such a shell is said to be an elliptic membrane shell if the following two additional assumptions are satisfied: first, $\gamma_{0}=\gamma$, i.e., the homogeneous boundary condition of place is imposed over the entire lateral face $\gamma \times[-\varepsilon, \varepsilon]$ of the shell, and second, its middle surface $\boldsymbol{\theta}(\bar{\omega})$ is elliptic, according to the definition given in Section 2. Note that the assumption $\gamma_{0}=\gamma$ implies that the space $\boldsymbol{V}_{K}(\omega)$ introduced in Section 3 now reduces to

$$
\boldsymbol{V}_{K}(\omega)=H_{0}^{1}(\omega) \times H_{0}^{1}(\omega) \times H_{0}^{2}(\omega) .
$$

To begin with, we recall a crucial inequality that holds for elliptic surfaces (cf., e.g., Theorem 2.7-3 of [21]).

Theorem 7.1. Let $\omega$ be a domain in $\mathbb{R}^{2}$ and let $\boldsymbol{\theta} \in \mathcal{C}^{3}\left(\bar{\omega} ; \mathbb{E}^{3}\right)$ be an immersion such that $\boldsymbol{\theta}(\bar{\omega})$ is an elliptic surface. Define the space

$$
\boldsymbol{V}_{M}(\omega):=H_{0}^{1}(\omega) \times H_{0}^{1}(\omega) \times L^{2}(\omega),
$$

and the norm $\|\cdot\|_{\boldsymbol{V}_{M}(\omega)}$ by

$$
\|\boldsymbol{\eta}\|_{\boldsymbol{V}_{M}(\omega)}:=\left\{\sum_{\alpha}\left\|\eta_{\alpha}\right\|_{1, \omega}^{2}+\left\|\eta_{3}\right\|_{0, \omega}^{2}\right\}^{1 / 2} \quad \text { for each } \boldsymbol{\eta}=\left(\eta_{i}\right) \in \boldsymbol{V}_{M}(\omega) .
$$

Then there exists a constant $c=c(\omega, \boldsymbol{\theta})>0$ such that

$$
\|\boldsymbol{\eta}\|_{\boldsymbol{V}_{M}(\omega)} \leq c\left\{\sum_{\alpha, \beta}\left\|\gamma_{\alpha \beta}(\boldsymbol{\eta})\right\|_{0, \omega}^{2}\right\}^{1 / 2}
$$

for all $\boldsymbol{\eta}=\left(\eta_{i}\right) \in \boldsymbol{V}_{M}(\omega)$.

Prepared using sagej.cls 
A natural formulation of a set of time-dependent two-dimensional equations (again, "two-dimensional", in the sense that they are posed over the twodimensional subset $\omega$ ) can be derived in the same way as in Section 3.

Let us introduce the problem $\mathcal{P}_{M}^{\varepsilon}(\omega)$, describing the evolution of timedependent elliptic membrane shells.

Problem $\mathcal{P}_{M}^{\varepsilon}(\omega)$. Find a vector field $\boldsymbol{\zeta}^{\varepsilon}=\left(\zeta_{i}^{\varepsilon}\right):[0, T] \rightarrow \boldsymbol{V}_{M}(\omega)$ such that

$$
\boldsymbol{\zeta}^{\varepsilon} \in \mathcal{C}^{0}\left([0, T] ; \boldsymbol{V}_{M}(\omega)\right) \cap \mathcal{C}^{1}\left([0, T] ; \boldsymbol{L}^{2}(\omega)\right),
$$

that satisfies the following variational equations

$2 \varepsilon \rho \frac{\mathrm{d}^{2}}{\mathrm{~d} t^{2}} \int_{\omega} \zeta_{i}^{\varepsilon}(t) \eta_{i} \sqrt{a} \mathrm{~d} y+\varepsilon \int_{\omega} a^{\alpha \beta \sigma \tau} \gamma_{\sigma \tau}\left(\boldsymbol{\zeta}^{\varepsilon}(t)\right) \gamma_{\alpha \beta}(\boldsymbol{\eta}) \sqrt{a} \mathrm{~d} y=\int_{\omega} p^{i, \varepsilon}(t) \eta_{i} \sqrt{a} \mathrm{~d} y$, for all $\boldsymbol{\eta}=\left(\eta_{i}\right) \in \boldsymbol{V}_{M}(\omega)$, in the sense of distributions in $(0, T)$, and that satisfies the following initial conditions

$$
\left\{\begin{array}{l}
\zeta^{\varepsilon}(0)=\zeta_{0} \\
\dot{\zeta}^{\varepsilon}(0)=\zeta_{1}
\end{array}\right.
$$

where $\boldsymbol{\zeta}_{0} \in \boldsymbol{V}_{M}(\omega)$ and $\boldsymbol{\zeta}_{1} \in \boldsymbol{L}^{2}(\omega)$ are prescribed.

We say that $\boldsymbol{\zeta}^{\varepsilon}$ is a strong solution of Problem $\mathcal{P}_{M}^{\varepsilon}(\omega)$ if

$$
\boldsymbol{\zeta}^{\varepsilon} \in \mathcal{C}^{0}\left([0, T] ; \boldsymbol{V}_{M}(\omega)\right) \cap \mathcal{C}^{1}\left([0, T] ; \boldsymbol{L}^{2}(\omega)\right),
$$

if $\boldsymbol{\zeta}^{\varepsilon}$ satisfies the variational equations of Problem $\mathcal{P}_{M}^{\varepsilon}(\omega)$ in the sense of distributions in $(0, T)$, and also satisfies the initial conditions.

Since the space $\boldsymbol{V}_{M}(\omega)$ is continuously and densely embedded in the space $\boldsymbol{L}^{2}(\omega)$ and since, as a consequence of the uniform positive-definiteness of the elasticity tensor of the shell $\left(a^{\alpha \beta \sigma \tau}\right)$ and Theorem 7.1, the bilinear form $B_{M}: \boldsymbol{V}_{M}(\omega) \times \boldsymbol{V}_{M}(\omega) \rightarrow \mathbb{R}$ defined by

$$
B_{M}(\boldsymbol{\eta}, \boldsymbol{\xi}):=\varepsilon \int_{\omega} a^{\alpha \beta \sigma \tau} \gamma_{\sigma \tau}(\boldsymbol{\eta}) \gamma_{\alpha \beta}(\boldsymbol{\xi}) \sqrt{a} \mathrm{~d} y,
$$

is $\boldsymbol{V}_{M}(\omega)$-elliptic, the existence and uniqueness of strong solutions of Problem $\mathcal{P}_{M}^{\varepsilon}(\omega)$ is classical (cf, e.g., Theorem 8.2-2 of [31]).

Theorem 7.2. Problem $\mathcal{P}_{M}^{\varepsilon}(\omega)$ admits a unique strong solution $\boldsymbol{\zeta}^{\varepsilon} \in$ $\mathcal{C}^{0}\left([0, T] ; \boldsymbol{V}_{M}(\omega)\right) \cap \mathcal{C}^{1}\left([0, T] ; \boldsymbol{L}^{2}(\omega)\right)$.

\section{Acknowledgments}

The author expresses his sincere gratitude to the Reviewers for their valuable help and suggestions.

The author would like to express his gratitude to Professor Philippe G. Ciarlet for his encouragement and advice.

The author acknowledges partial support by the ERC advanced grant 668998 (OCLOC) under the EU's H2020 research program.

Prepared using sagej.cls 


\section{References}

[1] Huda MN, Jumat M and Islam A. Flexural performance of reinforced oil palm shell and palm oil clinker concrete (pscc) beam. Constr Bulld Mater 2016; 127: 18-25.

[2] Singh V, Mahapatra T and SK P. Nonlinear flexural analysis of single/doubly curved smart composite shell panels integrated with pfrc actuator. Eur J Mech A-Solid 2016; 60: 300-314.

[3] Kar V and Panda S. Nonlinear flexural vibration of shear deformable functionally graded spherical shell panel. Compos Struct 2015; 18(3): 693709.

[4] Totaro G. Flexural, torsional, and axial global stiffness properties of anisogrid lattice conical shells in composite material. Compos Struct 2016; 153: 738-745.

[5] Jayaprithika A and Sekar S. Stress-strain characteristics and flexural behaviour of reinforced eco-friendly coconut shell concrete. Constr Build Mater 2016; 117: 244-250.

[6] Ciarlet PG and Destuynder P. A justification of the two-dimensional linear plate model. J Mécanique 1979; 18: 315-344.

[7] Ciarlet PG and Miara B. Justification of a two-dimensional shallow shell model in linearized elasticity. C R Acad Sci Paris Sér I Math 1990; 311(9): $571-574$.

[8] Ciarlet PG and Miara B. Justification of the two-dimensional equations of a linearly elastic shallow shell. Comm Pure Appl Math 1992; 45(3): 327-360.

[9] Ciarlet PG and Lods V. On the ellipticity of linear membrane shell equations. J Math Pures Appl 1996; 75: 107-124.

[10] Ciarlet PG and Lods V. Asymptotic analysis of linearly elastic shells. I. Justification of membrane shell equations. Arch Rational Mech Anal 1996; 136(2): 119-161.

[11] Ciarlet PG and Lods V. Asymptotic analysis of linearly elastic shells. III. Justification of Koiter's shell equations. Arch Rational Mech Anal 1996; 136(2): 191-200.

[12] Ciarlet PG and Lods V. Asymptotic analysis of linearly elastic shells: "generalized membrane shells". J Elasticity 1996; 43(2): 147-188.

[13] Ciarlet PG, Lods V and Miara B. Asymptotic analysis of linearly elastic shells. II. Justification of flexural shell equations. Arch Rational Mech Anal 1996; 136(2): 163-190.

Prepared using sagej.cls 
[14] Ciarlet PG, Mardare C and Shen X. Donati compatibility conditions for membrane and flexural shells. Anal Appl 2015; 13(6): 685-705.

[15] Ciarlet PG, Mardare C and Piersanti P. An obstacle problem for elliptic membrane shells. Math Mech Solids 2019; 24(5): 1503-1529.

[16] Ciarlet PG, Mardare C and Piersanti P. Un problème de confinement pour une coque membranaire linéairement élastique de type elliptique. CR Acad Sci Paris, Sér I 2018; 356(10): 1040-1051.

[17] Ciarlet PG and Piersanti P. A confinement problem for a linearly elastic Koiter's shell. CR Acad Sci Paris, Sér I 2019; 357: 221-230.

[18] Ciarlet PG and Piersanti P. An obstacle problem for Koiter's shells. Math Mech Solids ; DOI:10.1177/1081286519825979.

[19] Xiao L. Asymptotic analysis of dynamic problems for linearly elastic shellsjustification of equations for dynamic flexural shells. Chinese Ann Math Ser B 2001; 22(3): 13-22.

[20] Xiao L. Asymptotic analysis of dynamic problems for linearly elastic shellsjustification of equations for dynamic Koiter shells. Chinese Ann Math Ser B 2001; 22(3): 267-274.

[21] Ciarlet PG. Mathematical Elasticity. Vol. III: Theory of Shells. Amsterdam: North-Holland, 2000.

[22] Ciarlet PG. An Introduction to Differential Geometry with Applications to Elasticity. Dordrecht: Springer, 2005.

[23] Leoni G. A First Course in Sobolev Spaces, Graduate Studies in Mathematics, volume 181. Second ed. Providence: American Mathematical Society, 2017.

[24] Ciarlet PG. Mathematical Elasticity. Vol. I: Three-Dimensional Elasticity. Amsterdam: North-Holland, 1988.

[25] Koiter WT. On the foundations of the linear theory of thin elastic shells. I, II. Nederl Akad Wetensch Proc Ser B 73 (1970), 169-182; ibid 1970; 73: $183-195$.

[26] Bernadou M and Ciarlet PG. Sur l'ellipticité du modèle linéaire de coques de W. T. Koiter. Computing Methods in Applied Sciences and Engineering, Part 1 1996; 134: pp. 89-136.

[27] Bernadou M, Ciarlet PG and Miara B. Existence theorems for twodimensional linear shell theories. J Elasticity 1994; 34: 111-138.

[28] Gronwall TH. Note on the derivatives with respect to a parameter of the solutions of a system of differential equations. Ann of Math 1919; 20(4): 292-296.

Prepared using sagej.cls 
[29] Hartman P. Ordinary Differential Equations. Second ed. Philadelphia: Society for Industrial and Applied Mathematics, 1982.

[30] Lions JL. Quelques méthodes de résolution des problèmes aux limites non linéaires. Dunod; Gauthier-Villars, Paris, 1969.

[31] Raviart $\mathrm{P}$ and Thomas J. Introduction à l'Analyse Numérique des Équations aux Dérivées Partielles. Paris: Dunod, 1988.

[32] Brezzi F and Fortin M. Mixed and Hybrid Finite Element Methods. New York: Springer, 1991.

[33] Ciarlet PG. Introduction to Numerical Linear Algebra and Optimisation. Cambridge: Cambridge University Press, 1989.

[34] Ciarlet PG. Linear and Nonlinear Functional Analysis with Applications. Philadelphia: Society for Industrial and Applied Mathematics, 2013.

[35] Kyritsi-Yiallourou S and Papageorgiou NS. Handbook of Applied Analysis. New York: Springer, 2009.

[36] Evans LC. Partial Differential Equations. Second ed. Providence: American Mathematical Society, 2010.

Prepared using sagej.cls 